ARTICLE

\title{
Comprehensive functional analysis of the tousled-like kinase 2 frequently amplified in aggressive luminal breast cancers
}

Jin-Ah Kim 1,2,3, Ying Tan1,2,3, Xian Wang1,2,3,4,5, Xixi Cao 1,2,3, Jamunarani Veeraraghavan¹,2,3, Yulong Liang6, Dean P. Edwards ${ }^{2,7,8}$, Shixia Huang ${ }^{2,8}$, Xuewen Pan ${ }^{9}$, Kaiyi Li ${ }^{6}$, Rachel Schiff1,2,3,8 \& Xiao-Song Wang ${ }^{1,2,3,4,5,8}$

More aggressive and therapy-resistant oestrogen receptor (ER)-positive breast cancers remain a great clinical challenge. Here our integrative genomic analysis identifies tousled-like kinase 2 (TLK2) as a candidate kinase target frequently amplified in $\sim 10.5 \%$ of ER-positive breast tumours. The resulting overexpression of TLK2 is more significant in aggressive and advanced tumours, and correlates with worse clinical outcome regardless of endocrine therapy. Ectopic expression of TLK2 leads to enhanced aggressiveness in breast cancer cells, which may involve the EGFR/SRC/FAK signalling. Conversely, TLK2 inhibition selectively inhibits the growth of TLK2-high breast cancer cells, downregulates ER $\alpha, B C L 2$ and SKP2, impairs G1/S cell cycle progression, induces apoptosis and significantly improves progression-free survival in vivo. We identify two potential TLK2 inhibitors that could serve as backbones for future drug development. Together, amplification of the cell cycle kinase TLK2 presents an attractive genomic target for aggressive ER-positive breast cancers.

\footnotetext{
${ }^{1}$ Lester \& Sue Smith Breast Center, Baylor College of Medicine, Houston, Texas 77030, USA. ${ }^{2}$ Dan L. Duncan Cancer Center, Baylor College of Medicine, Houston, Texas 77030, USA. ${ }^{3}$ Department of Medicine, Baylor College of Medicine, Houston, Texas 77030, USA. ${ }^{4}$ University of Pittsburgh Cancer Institute, University of Pittsburgh, Pittsburgh, Pennsylvania 15213, USA. ${ }^{5}$ Department of Pathology, University of Pittsburgh, Pittsburgh, Pennsylvania 15213, USA. ${ }^{6}$ Department of Surgery, Baylor College of Medicine, Houston, Texas 77030, USA. ${ }^{7}$ Department of Pathology \& Immunology, Baylor College of Medicine, Houston, Texas 77030, USA. ${ }^{8}$ Department of Molecular and Cellular Biology, Baylor College of Medicine, Houston, Texas 77030, USA. ${ }^{9}$ Department of Biochemistry and Molecular Biology, Baylor College of Medicine, Houston, Texas 77030, USA. Correspondence and requests for materials should be addressed to X.-S.W. (email: wangx13@pitt.edu).
} 
vast majority of breast cancers express the oestrogen receptor $(\mathrm{ER}+)$ and can be treated with endocrine therapy; however, the clinical outcome varies radically between different patients. ER + breast cancers are also known as luminal breast cancers and can be subdivided into $\mathrm{A}$ and $\mathrm{B}$ subtypes. The luminal B tumours are more aggressive ER+ breast cancers characterized by poorer tumour grade, larger tumour size and higher proliferation index. Clinically, such tumours are prone to develop endocrine resistance, which poses a great challenge to clinical management. Identifying the genetic aberrations underlying the enhanced aggressiveness of these tumours, and developing effective therapeutic strategies to target them, are in high demand. Recent prominent success of the CDK4/6-specific inhibitors in clinical trials for advanced breast cancers have attracted wide-spread attention to the potential of cell cycle kinases as viable drug targets in breast cancer $^{1}$. Thus, discovering new cell cycle kinase targets that can tackle the more aggressive ER + breast cancers will be of critical clinical significance.

Genomic amplifications lead to deregulations of oncogenes to which cancer cells become often addicted in specific tumours. Such events, however, usually affect a large number of genes in cancer genomes, which make it difficult to identify the primary oncogene targets of these amplifications. In our previous study, we discovered that cancer genes possess distinctive yet complicated 'gene concept signature', which include cancer-related signalling pathways, molecular interactions, transcriptional motifs, protein domains and gene ontologies ${ }^{2}$. Based on this observation, we developed a Concept Signature (or ConSig) analysis that prioritizes the biological importance of candidate genes underlying cancer via computing their strength of association with those cancer-related signature concepts (http://consig.cagenome.org) ${ }^{2-4}$. In our previous study, we have applied this analysis to reveal the primary target genes of chromosome $17 \mathrm{q}$ amplifications in breast cancer ${ }^{5}$. Here we postulate that the ConSig analysis may be used to effectively nominate dominantly acting cancer genes from the genomic amplifications in cancer at a genome-wide scale, which can be further translated into viable therapeutic targets by interrogating pharmacological databases (Fig. 1a). Toward this end, we have assembled a genome-wide analysis called 'ConSig-Amp' to discover viable therapeutic targets in cancer from multi-dimensional genomic data sets.

Applying this analysis to the genomic data from The Cancer Genome Atlas (TCGA) nominated a new oncogene target called tousled-like kinase 2 (TLK2) frequently amplified in aggressive luminal breast cancer. Tousled-like kinases (TLKs) are nuclear serine/threonine kinases that promote chromatin assembly during S-phase and also chromosome segregation during mitosis ${ }^{6-8}$. The TLK gene family includes two members, TLK1 and TLK2 (Supplementary Fig. 1a) ${ }^{9}$. Most, if not all, of the reports about the function of TLKs focus on the study of TLK1, while the function of TLK2 and its role in human cancers are still largely unknown. To date, there is no functional characterization of TLK2 in breast cancer, although TLK2 single nucleotide polymorphism has been associated with increased breast cancer risk ${ }^{10}$, and most recently TLK2 has been reported as an amplicon-associated highly phosphorylated kinase in luminal breast cancer ${ }^{11}$. Here we discovered that TLK2 overexpression endows enhanced invasiveness of luminal breast cancers, and appears to be addictive for TLK2-amplified breast cancers so that TLK2 inhibition renders decreased cancer cell viability and increased apoptosis. This suggests that TLK2 may serve as an attractive genomic target for the aggressive luminal breast cancers harbouring TLK2 amplifications.

\section{Results}

TLK2 as a lead target amplified in ER + breast cancers. To systematically reveal new therapeutic targets, we applied the 'ConSig-Amp' analysis to the genomic data sets for breast cancers from TCGA ${ }^{12}$. First, we identified all human genes that are amplified in $>5 \%$ of ER + breast cancers based on copy-number data. Candidates were then benchmarked with the ConSig-Amp score which is calculated by multiplying ConSig score (Methods) by the correlation between gene expression and copy number, to prioritize biologically important targets that are upregulated by genomic amplifications. Potentially druggable candidates were then selected according to a drug-target database compiled from multiple sources ${ }^{13-15}$. This revealed several known kinase targets in breast cancer such as ERBB2, PAK1, RPS6KB1 and PTK2 (refs 16,17), together with a new candidate kinase target, TLK2 (Fig. 1b; Supplementary Table 1).

Copy-number data from TCGA show that TLK2 is amplified in about $\sim 9 \%$ of all breast cancers (Fig. 1c), and such events are more frequent in ER-positive than negative breast cancers (10.5 versus $2.9 \%$ ) (Supplementary Fig. $1 \mathrm{~b})$. TLK2 locates in a frequently amplified region in chr17q23.2 close to RPS6KB1 amplifications, and is far apart from the ERBB2 amplifications in 17q12 (Supplementary Fig. 2). Thus TLK2 is not co-amplified with ERBB2. Supplementary Figure 3a, b shows the copy-number data of TLK2 and known amplified oncogenes in breast cancer in TCGA $^{12}$ and Metabric ${ }^{18}$ data sets. TLK2 copy number does not correlate with most known oncogene amplifications, except RPSKB1 (Pearson $R=0.796$ for the TCGA data set and $R=0.768$ for the Metabric data set). While Her 2 amplifications are enriched in TLK2-amplified tumours, their copy numbers do not correlate with each other $(R=0.187$ for TCGA data set and $R=0.201$ for the Metabric data set). This suggests that TLK2 is frequently co-amplified with RPS6KB1, but not with other known amplified genes in breast cancer such as ERBB2, MYC, CCND1, and so on. Gene expression data show that TLK2 expression is primarily upregulated by copy-number increase at this locus (Fig. 1d, $R=0.81$ ), and correlates with increased tumour stage (Fig. 1e). Among all the breast cancer subtypes, luminal B breast cancers most frequently harbour TLK2 amplifications (21.3\%) (Supplementary Fig. 1b), and also present the highest TLK2 expression level (Fig. 1e). Here, the Luminal B subtyping is based on the 50 -gene PAM50 predictor $^{19}$ using Agilent gene expression data, and is provided by TCGA ${ }^{12}$.

TLK2 overexpression correlates with worse clinical outcome. To examine the prognostic effect of TLK2 overexpression (OE), we analysed the available survival data for TCGA breast cancer patients and compared the group of patients with TLK2-high tumours versus the rest (see Methods). This revealed a significantly worse overall survival of the TLK2-high group (based on log-rank test, $P=0.040)$. TLK2 is mostly amplified in $\mathrm{ER}+$ breast cancers, which are commonly treated with endocrine therapy. To examine if endocrine therapy can eliminate the prognostic effect of TLK2 OE, we analysed the gene expression data set by Loi et al. ${ }^{20}$ for $263 \mathrm{ER}+$ breast tumours treated with adjuvant tamoxifen monotherapy, which revealed a significantly worse recurrence-free survival of patients with TLK2-high tumours (based on log-rank test, $P=0.001$ ). To further corroborate this finding, we analysed a large gene expression data set for breast cancers with treatment and prognostic information (the Metabric data set) ${ }^{18}$ (Fig. 1f). Among the 220 $\mathrm{ER}+$ breast cancer patients with no adjuvant treatment, those with TLK2-high tumours showed significantly worse disease-specific survival than the rest of the patients (based on log-rank test, $P=0.012$ ). The prognostic effect is preserved in the 
a

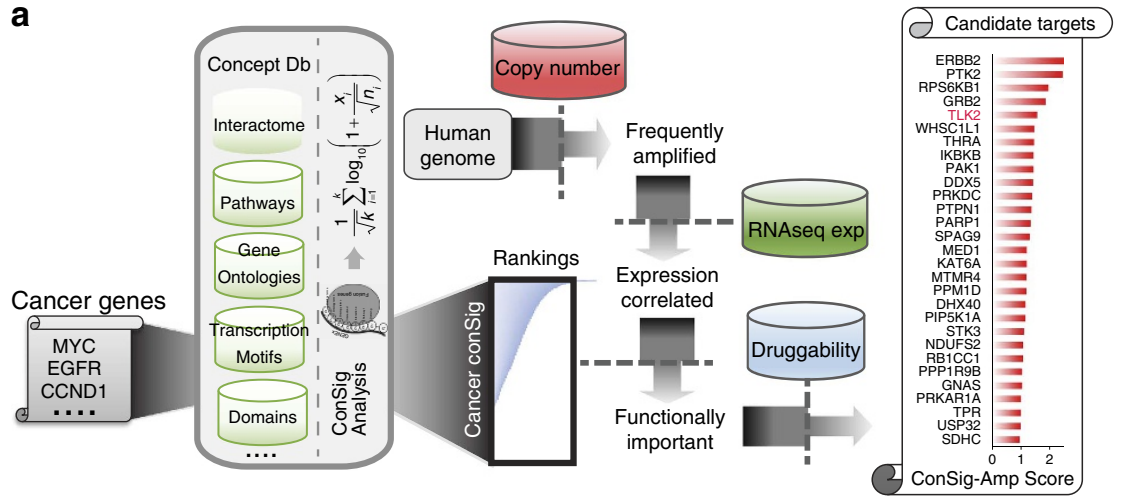

C

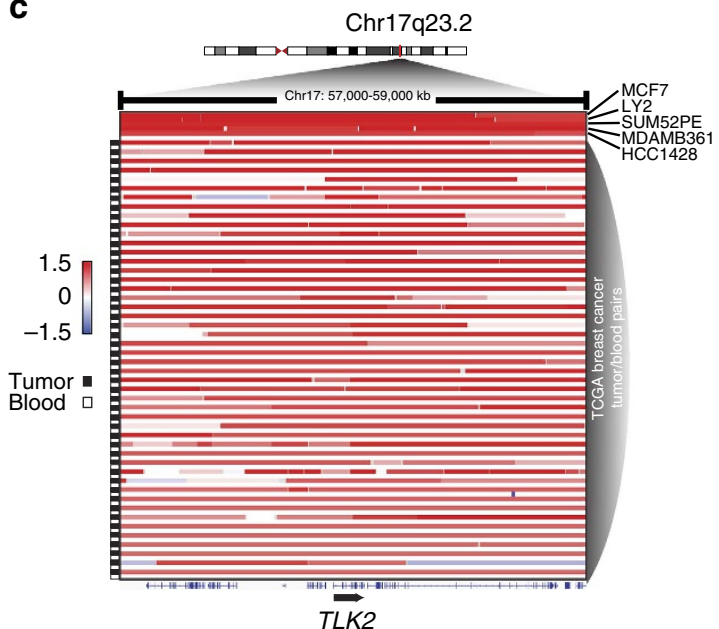

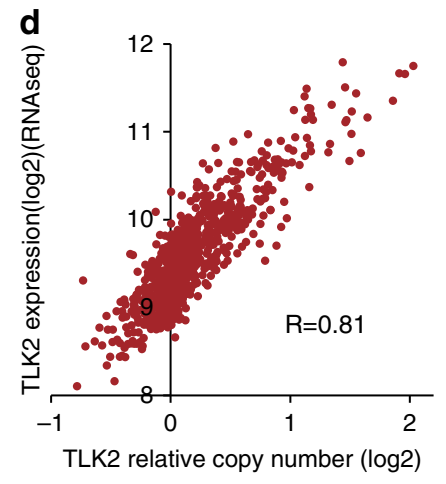

b

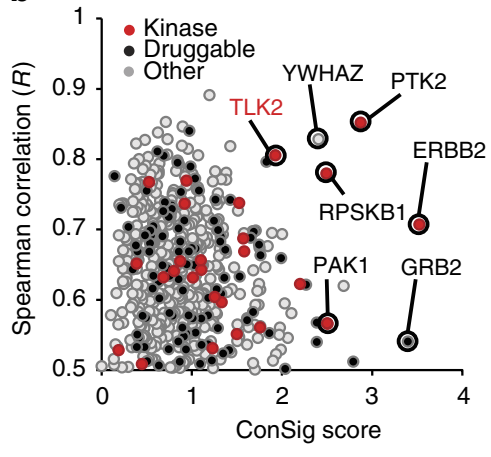

$\mathbf{f}$

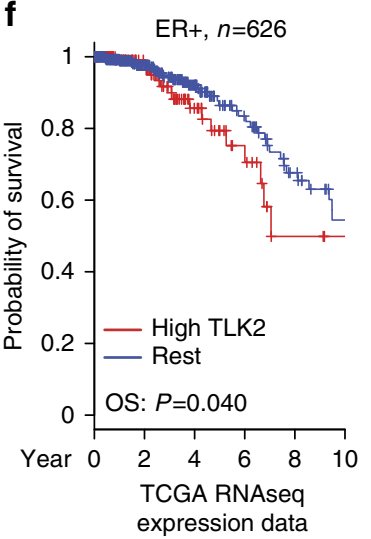

ER+/Tam, $n=263$

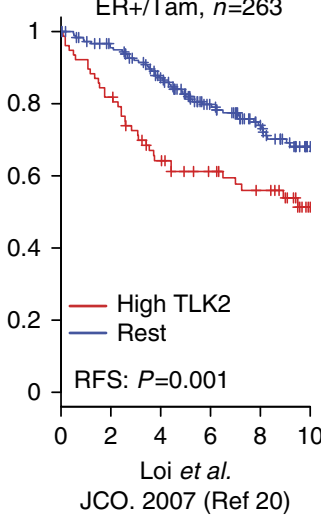

ER+/Unt, $n=219$

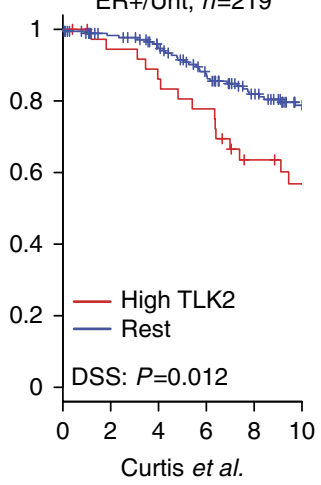

Nature 2012 (Ref 18)

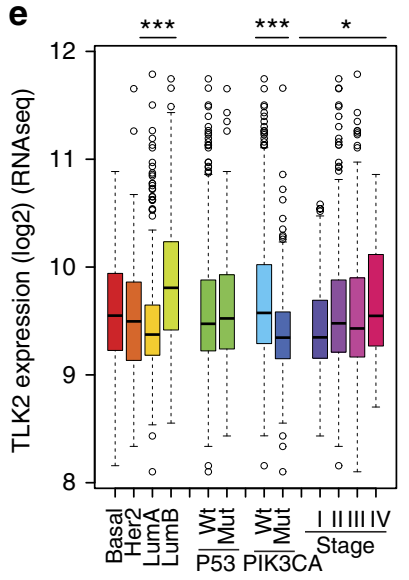

Figure 1 | ConSig-Amp identifies TLK2 as a candidate druggable target frequently amplified in breast cancer. (a) The bioinformatics workflow of ConSig-Amp to discover therapeutically relevant oncogene targets in cancer at genome-wide scale based on copy-number and RNAseq data sets. The ConSig-Amp score is calculated by multiplying the ConSig score (see Methods) with the correlation between gene expression and copy number.

(b) Prioritizing amplified breast cancer oncogene targets by ConSig score and Spearman's correlation between copy number (Affymetrix SNP 6.0 array) and gene expression (RNAseq). Data shown here are from TCGA. (c) Representative copy-number data showing amplifications at the TLK2 locus in paired breast tumour and peripheral blood (data from TCGA ${ }^{52}$ ), or breast cancer cell lines (data from Heiser et al. ${ }^{21}$ ). This figure is based on Affymetrix SNP 6.0 array data annotated with genome build hg18. Positive cell line or tumour samples are sorted based on the level of TLK2 amplifications, and the structures of genes involved in the presented region are shown under the illustration. (d) TLK2 expression (based on RNAseq data) is primarily regulated by gene copy number (based on Affymetrix SNP 6.0 array data). The Spearman's correlation is $R=0.81$. (e) TLK2 expression in different breast cancer subtypes based on RNAseq data. Copy number and RNAseq expression data shown in $\mathbf{d}, \mathbf{e}$ are from TCGA. The whiskers indicate the max and min values (excluding outliers) and horizontal lines represent the 1st, 2 nd and 3rd quartiles. ${ }^{\star} P<0.05 ;{ }^{\star \star \star} P<0.001$. (f) Kaplan-Meier plots based on multiple gene expression data sets showing correlation of TLK2 overexpression with the outcome of systemically untreated or endocrine-treated ER + breast cancer patients. HT, hormone treated; Tam, tamoxifen-treated; Unt, untreated. $P$ values are calculated based on log-rank tests.

389 ER + breast cancer patients treated with endocrine monotherapy (based on log-rank test, $P=0.012$ ). Of note, about $93 \%$ of the untreated and endocrine-treated ER+ tumours of the Metabric data set are Her2 negative. These data support a poorer outcome of patients with TLK2-high tumours irrespective of endocrine therapy. 
Phenotypic changes following ectopic expression of TLK2. To assess TLK2 expression levels in breast cancer cell lines and benign breast epithelial cells, we analysed the Affymetrix exon 1.0 ST expression array data from a previous study ${ }^{21}$. High-TLK2 expression was observed in multiple breast cancer cell lines (particularly $\mathrm{ER}+/ \mathrm{Her} 2-$ lines), but not in benign breast epithelial cells (Fig. 2a). This result was further verified through western blot analysis of a subset of these cell lines (Supplementary Fig. 4a). To determine the transforming activity of TLK2, we selected the MCF10A benign breast epithelial cell line and the TLK2-low luminal breast cancer cell line T47D (ER +/Her2 - ) for the engineering of TLK2 ectopic OE models. To achieve tuned TLK2 OE, we engineered the coding region of TLK2 into a doxycycline-inducible lentiviral vector, which was then transduced into these two cell lines (Figs $2 \mathrm{~b}$ and $3 \mathrm{a}$ ). Following 2 weeks of TLK2 induction, MCF10A or T47D cells expressing TLK2 did not show significant increase in clonogenic growth compared with the control cells (Figs $2 \mathrm{c}$ and $3 \mathrm{~b}$ ). However, soft-agar colony formation assays revealed significantly increased anchorage-independent growth after TLK2 overexpression in T47D cells (Fig. 3c).

To test if TLK2 may enhance cell invasiveness, we gauged the cell motility and invasion capability after TLK2 overexpression using transwell migration and invasion assays. Interestingly, inducible TLK2 overexpression in MCF10A or T47D cells strongly enhanced the cell migration and invasion capabilities in a dose-dependent manner (Figs $2 \mathrm{~d}$ and $3 \mathrm{~d}$ ). To attribute this to the excess of TLK2 protein, we abolished TLK2 expression by withdrawing the Dox induction in these cell models (Figs 2d and $3 \mathrm{~d}$ ). This eradicated the increased migration and invasion capabilities in both lines, suggesting the dependence of these properties on TLK2 expression itself. These data suggest the role of TLK2 in augmenting cell invasiveness. To examine the cell signalling changes following TLK2 overexpression in the T47D breast cancer cells, we performed western blot analysis of an array of signalling molecules in breast cancer. Interestingly, while the canonical growth factor signalling molecules in breast cancer such as AKT and ERK were not activated with TLK2 overexpression,

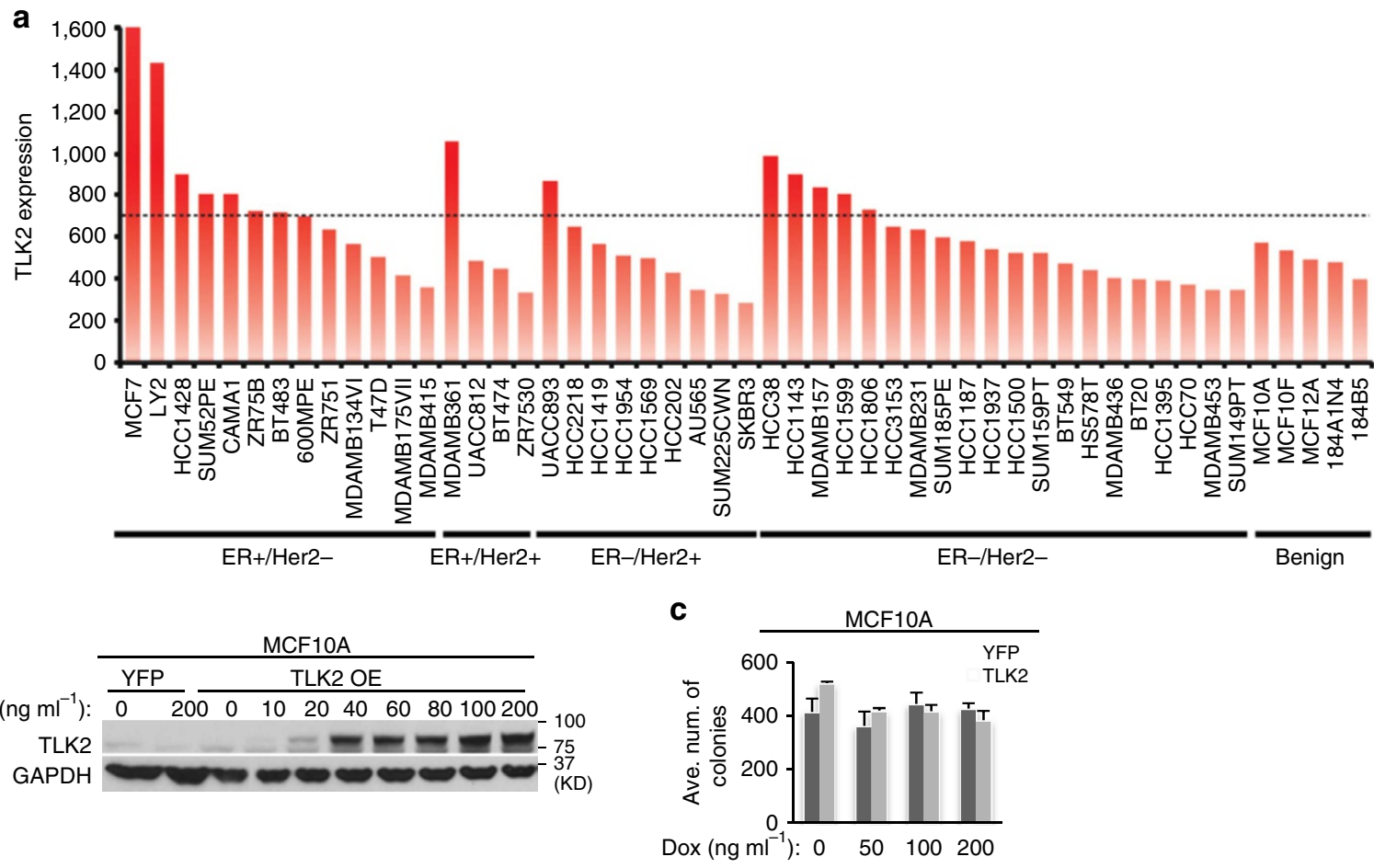

d MCF10A-TLK2
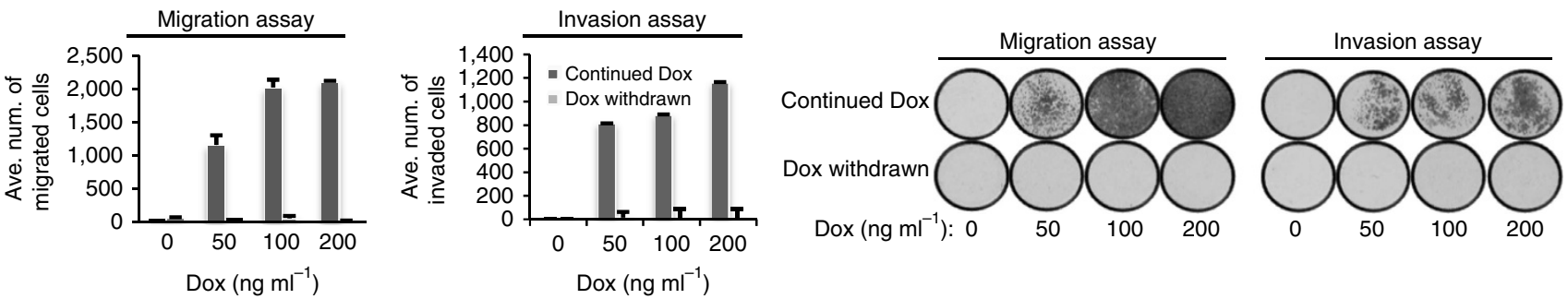

Figure 2 | The phenotypic changes after inducible ectopic expression of TLK2 in the MCF10A benign breast epithelial cells. (a) Bar chart showing TLK2 gene expression in a panel of breast cancer and benign cell lines based on exon expression array data ${ }^{21}$. (b) Western blot detecting the TLK2 proteins ectopically expressed in MCF10A cells after induction with different doses of doxycycline (Dox). (c) Cell survival following induction of TLK2 expression in MCF10A cells was measured by clonogenic assay. Error bars represent the s.d. of three replicate measurements per condition. (d) Transwell migration and matrigel invasion assays. After 2-week induction of TLK2 expression with different doses of Dox, Dox was either continued or withdrawn to test if the increased cell migration and invasion is dependent on TLK2 expression. Error bars represent the s.d. of three replicate measurements per condition. 
markedly increased phosphorylation of SRC on Y416 in the activation loop of its kinase domain, as well as modest increased activating phosphorylation of FAK and p38 were observed in the T47D cells overexpressing TLK2 (Fig. 3e). In addition, increased EGFR protein level and phosphorylation at the activating sites
(Y845 and Y1068) as well as increased Her2 auto-phosphorylation (Y1248 and Y1221/1222) are observed with TLK2 overexpression (Fig. 3e). Besides these changes, SKP2 protein level was also markedly elevated, and we will discuss later the significance of this alternation. SRC has a key role in breast

\section{a}

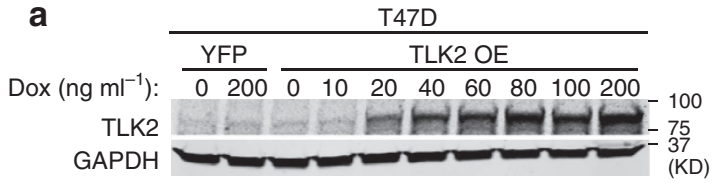

b
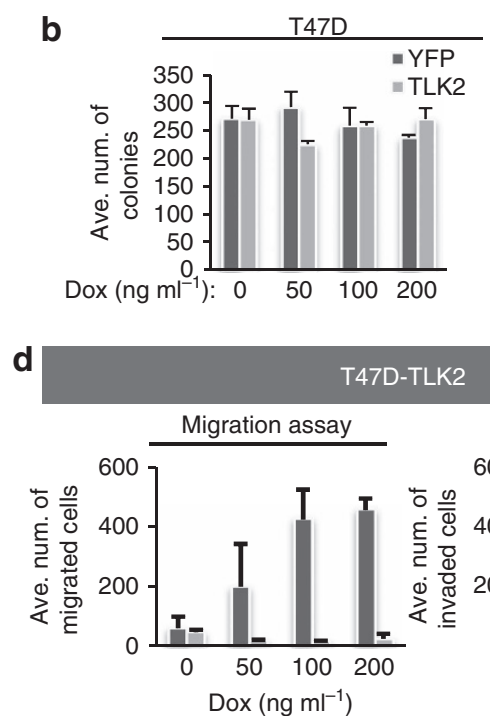

Dox $\left(\mathrm{ng} \mathrm{ml}^{-1}\right)$ : 0

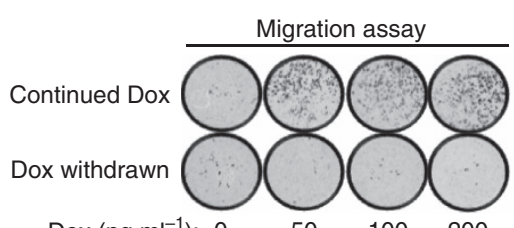

C
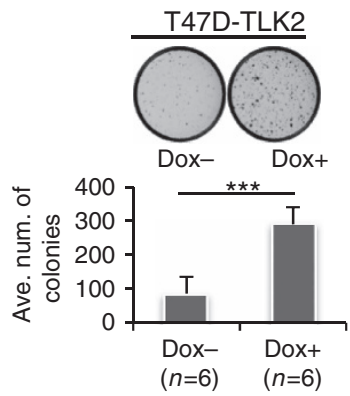
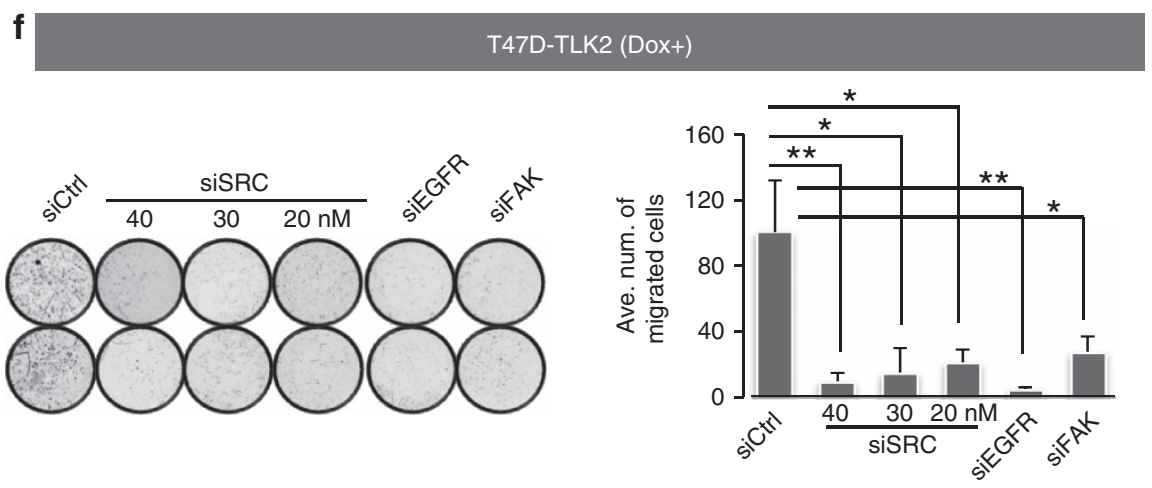

g

\section{MCF7-TLK2}
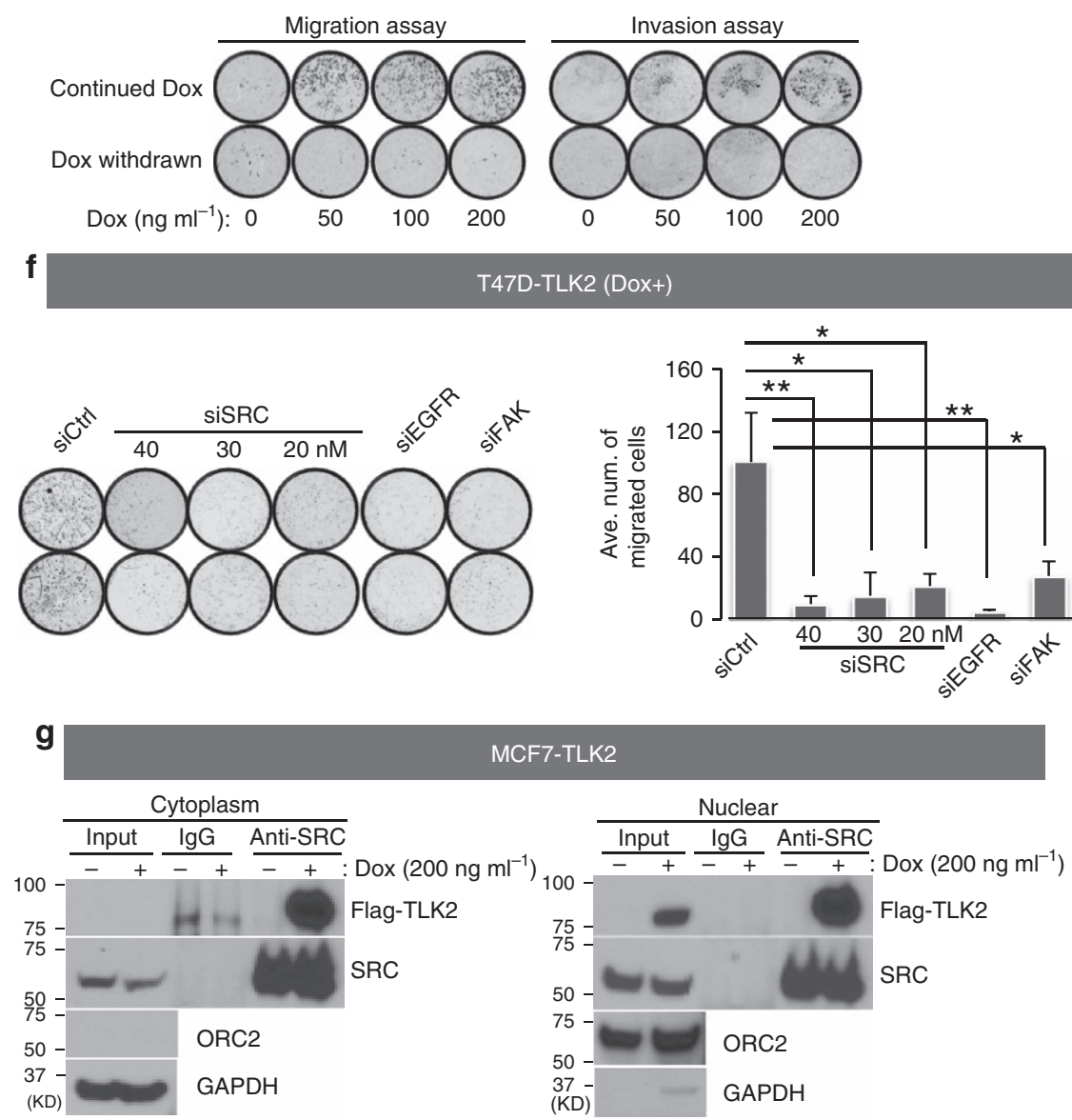

e

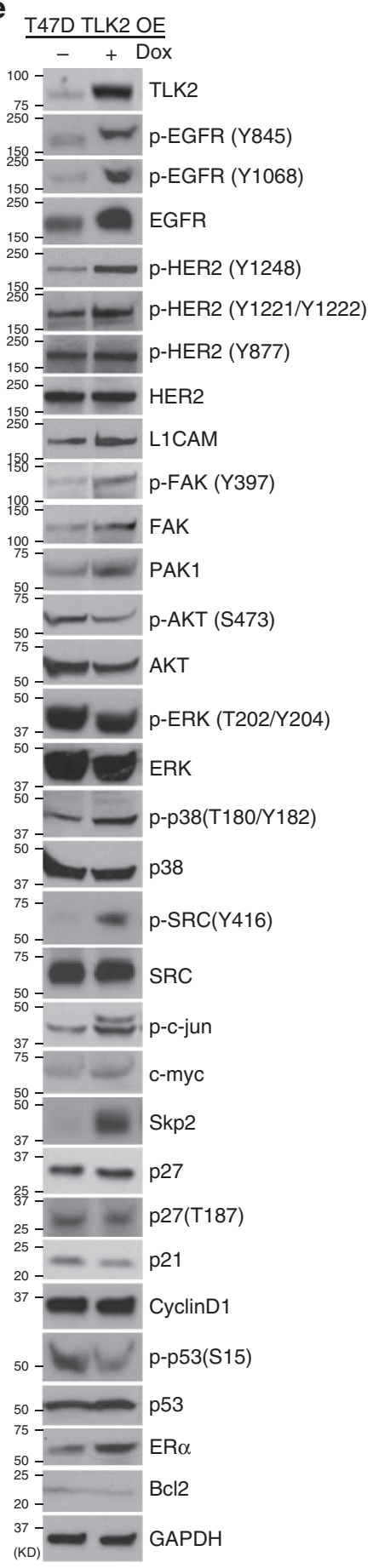


cancer cell migration, invasion and metastasis ${ }^{22,23}$, and activation of the FAK-SRC complex is known to mediate EGF-induced cell motility ${ }^{24,25}$. This suggests the possible involvement of the EGFR/SRC/FAK axis in the enhanced invasiveness driven by TLK2.

To access the significance of SRC, FAK and EGFR in TLK2-driven cell motility, we silenced SRC, FAK or EGFR using established siRNAs ${ }^{26-28}$, and assessed transwell cell migration. Interestingly, silencing of either SRC, FAK or EGFR in TLK2-overexpressing T47D cells significantly diminished the cell migration, suggesting that the EGFR/SRC/FAK axis may be involved in the enhanced invasiveness driven by TLK2 (Fig. 3f; Supplementary Fig. 5). To examine if TLK2 interacts with SRC, we performed immunoprecipitation (IP) of cytoplasm or nuclear fractions of the MCF7 cells expressing FLAG-tagged TLK2 using an established monoclonal antibody against SRC ${ }^{29,30}$, and detected the presence of TLK2 in SRC complex with anti-FLAG antibody. As a result, we observed the co-precipitation of TLK2 with SRC in both nuclear and cytoplasmic fractions (Fig. 3g). Taken together, these data suggest that EGFR/SRC/FAK axis may be important in TLK2-driven invasiveness in breast cancer cells, and TLK2 may engage this axis via interaction with SRC.

TLK2 silencing selectively inhibits TLK2-high cancer cells. Next, we went on to examine the effect of TLK2 silencing on breast cancer cell growth. Among the TLK2-high breast cancer cell lines, MCF7 and MDAMB361 show the highest TLK2 expression level (Fig. 2a), and also harbour high levels of TLK2 amplifications (Fig. 1c). MCF7 and MDAMB361 are both $\mathrm{ER}+$ luminal breast cancer cells, and are negative or positive for Her2, respectively. Thus these two cell lines will be ideal to study the effect of TLK2 inhibition in TLK2-amplified luminal breast cancer cells with different Her2 status. To affirm the TLK2 amplifications in these two cell lines, we performed fluorescence in situ hybridization (FISH) using a TLK2-specific probe and a centromere 17 probe (CEN17). As a result, the FISH assay verified TLK2 amplifications in the MCF7 and MDAMB361 cells with a TLK2:CEN17 ratio of 3.9 and 2.6, respectively (Supplementary Fig. 6). In addition to these TLK2-amplified breast cancer cell lines, we also selected a ER +/Her2 - luminal breast cancer cell line with moderately high TLK2 but without TLK2 amplification (CAMA1), a TLK2-low ER+/Her2luminal breast cancer (ZR75-1), and two benign epithelial cell lines (MCF12A and MCF10A). A MISSION esiRNA (Sigma) against TLK2 was then introduced into these selected cell lines, and the knockdown efficiency was verified by western blot (Supplementary Fig. 4b, c). The esiRNA is an endoribonuclease prepared siRNA pool that can deliver highly specific and effective gene silencing with lower off-target effects than single or pooled siRNAs ${ }^{31}$. In addition, a TLK2 siRNA\#1 targeting a different region from that of the esiRNA was also selected and verified as above (Supplementary Fig. 4b, c). The specificity of these siRNAs against TLK2 but not its paralogue TLK1 was verified by quantitative PCR and western blot (Supplementary Fig. 7a, b). MTT cell proliferation assays revealed that specific silencing of TLK2 by esiRNA or siRNA potently inhibited the growth of TLK2-high breast cancer cells (MCF7, MDAMB361 and CAMA1), but not that of the TLK2-low luminal ZR75-1 breast cancer cells or MCF12A and MCF10A benign breast epithelial cells (Fig. 4a). Interestingly, the MCF7 derivative strains with acquired resistance to tamoxifen (Tam-R) or oestrogen deprivation $(\mathrm{ED}-\mathrm{R})^{32}$ retained high sensitivity to TLK2 inhibition (Fig. 4a), suggesting the potential of TLK2 inhibition in managing these acquired resistant breast tumours. In addition, TLK2 inhibition by both TLK2 esiRNA and siRNA significantly repressed the migration of MCF7 and MDAMB361 cells (Fig. 4b), consistent with our reverse observations in the TLK2 overexpression models.

Inducible TLK2 inhibition suppresses clonogenic growth. Next, we engineered the MCF7 and MDAMB361 cell lines to inducibly express TLK2 shRNA targeting a region different from those of the TLK2 esiRNA and siRNA (Supplementary Fig. 4b, d), which allowed us to observe the long-term effects of TLK2 inhibition. With induction of TLK2 inhibition, decreased colony-forming ability was observed only in the TLK2-high MCF7 and MDAMB361 luminal breast cancer cells, but not in the TLK2-low T47D luminal breast cancer cells, as shown by clonogenic assays (Fig. 4c). In addition, we also observed potent inhibition of anchorage-independent growth following TLK2 inhibition in both MCF7 and MDAMB361 cells as shown by soft-agar colony formation assay (Fig. 4d).

To verify the specificity of this TLK2 shRNA, we engineered the MCF7 cells to inducibly express the TLK2 ORF with multiple silent mutations at the shRNA targeting sites. We then designed a TLK2 siRNA\#2 with the same target sequence as the TLK2 shRNA, and introduced it into the MCF7 cells inducibly expressing the mutated TLK2 ORF, which are subjected to clonogenic assays with or without Dox induction. Ectopic expression of TLK2 resulted in a significant rescue of the knockdown effect by TLK2 siRNA\#2 in a dose-dependent manner, which validates the specificity of the TLK2 shRNA (Fig. 4e).

The effect of TLK2 inhibition in a xenograft tumour model. To examine the therapeutic effect of TLK2 inhibition in a preclinical

Figure 3 | The phenotypic and cell signalling changes after ectopic expression of TLK2 in the T47D ER + /Her2 - luminal breast cancer cells. (a) Western blot detecting TLK2 protein ectopically expressed in T47D cells after induction with different doses of Dox. (b) Cell survival following induction of TLK2 expression in T47D cells was measured by clonogenic assay. Error bars represent the s.d. of three replicate measurements per condition. (c) TLK2 overexpression significantly enhanced anchorage-independent growth of T47D cells. TLK2 was overexpressed in T47D by treating with $100 \mathrm{ng} \mathrm{ml}^{-1}$ Dox before the soft-agar assays were performed. Error bars represent the s.d. of three replicate measurements per condition. (d) Transwell migration and matrigel invasion assays. Following TLK2 induction for 2 weeks, Dox was either continued or withdrawn to test if the increased cell migration and invasion is dependent on TLK2 overexpression. Error bars represent the s.d. of two replicate measurements per condition. (e) Alterations of key signalling molecules in breast cancer were examined by Western blot following TLK2 overexpression in T47D. TLK2 was induced by $200 \mathrm{ng} \mathrm{ml}{ }^{-1}$ Dox for 2 weeks. Dox, doxycyclin. (f) Transwell migration assays following SRC, EGFR or FAK knockdown in T47D cells overexpressing TLK2. The indicated concentration of siRNAs against SRC or $20 \mathrm{nM}$ of siRNAs against EGFR or FAK were transfected for 3 days following induction of TLK2 expression in T47D cells for two weeks (200 $\mathrm{ng} \mathrm{ml}^{-1}$ Dox). Western blot validation of SRC, EGFR or FAK silencing in T47D cells overexpressing TLK2 was shown in Supplementary Fig. 5. Error bars represent the s.d. of two replicate measurements per condition. (g) Co-immunoprecipitation of TLK2 with SRC in engineered MCF7 cells inducibly expressing Flag-tagged TLK2. Engineered MCF7 cells was treated with $200 \mathrm{ng} \mathrm{ml}{ }^{-1}$ Dox for $48 \mathrm{~h}$ and nuclear or cytoplasmic proteins were purified following subcellular fractionation. IP was performed using an established monoclonal antibody against SRC after conjugation with agarose beads. Western blot was performed using an anti-Flag antibody to detect the presence of TLK2 in SRC complex. Dox, Doxycyclin. 
a

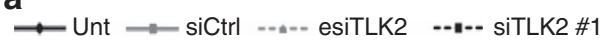
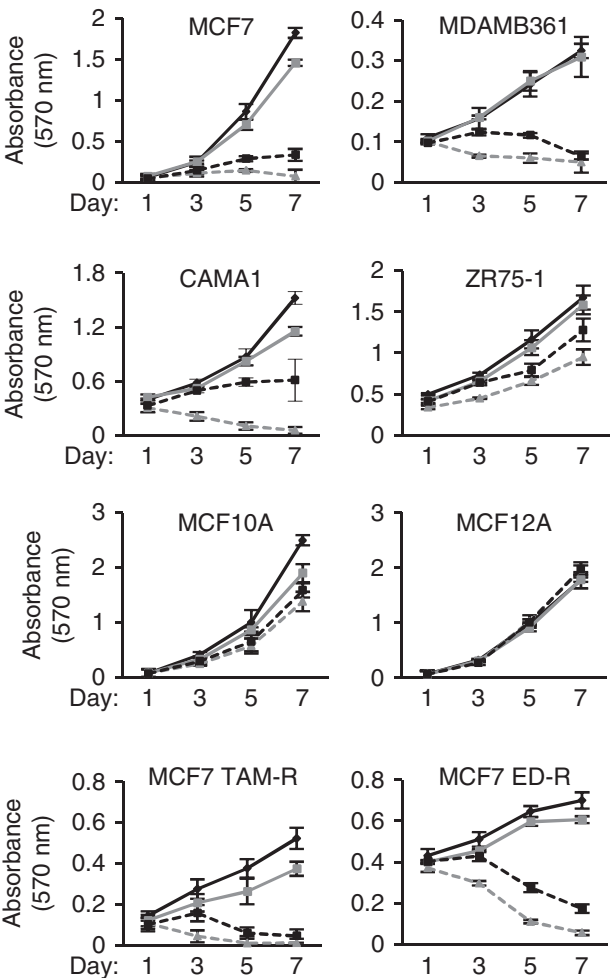

C
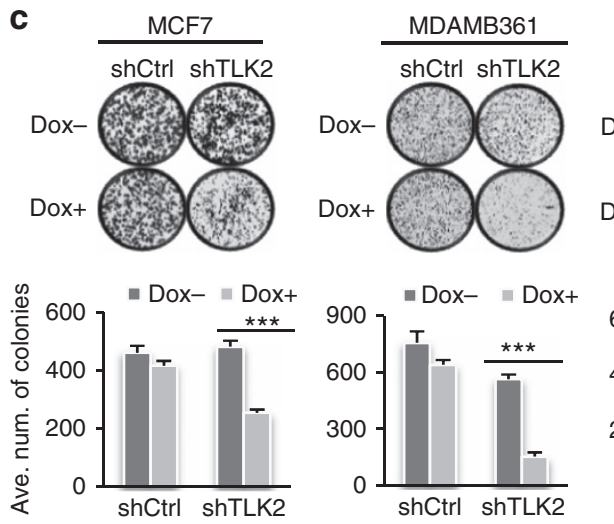

b
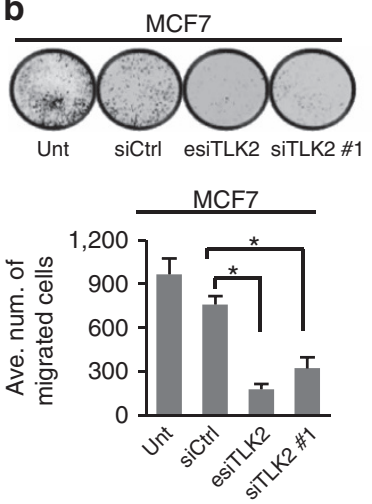

d
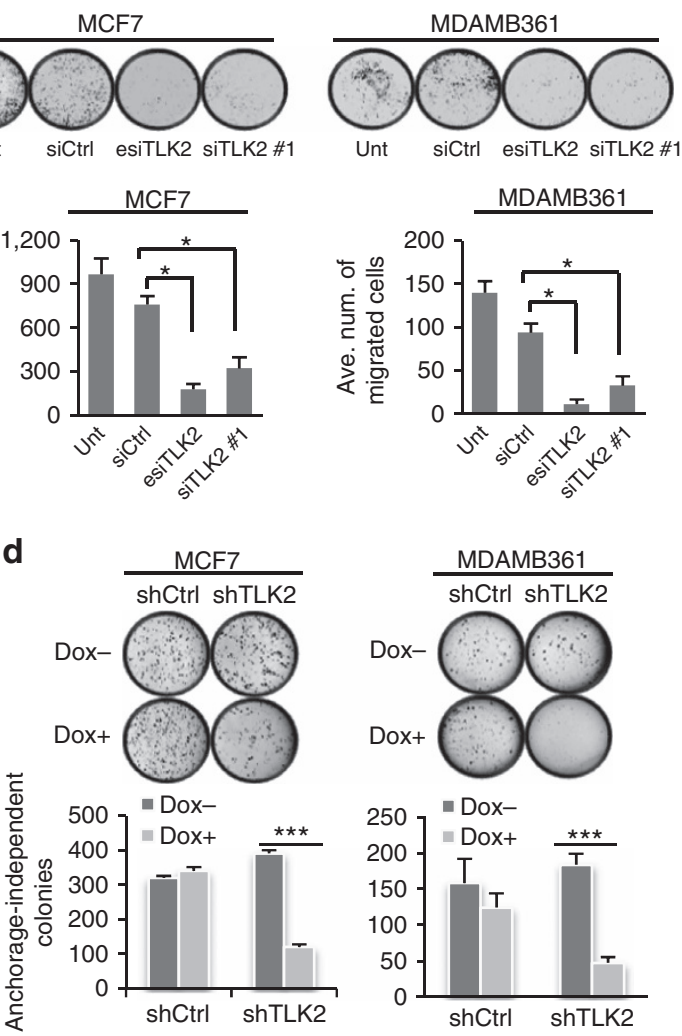

e
T47D

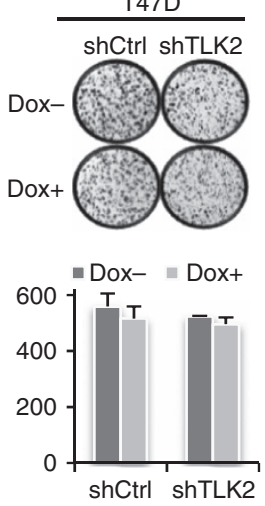

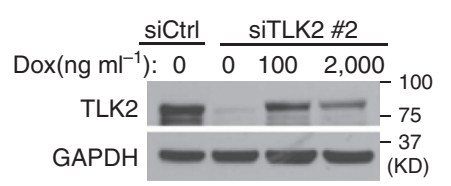

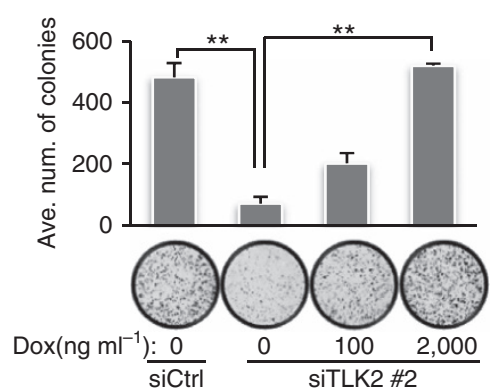

Figure 4 | The effect of TLK2 silencing in ER + breast cancer cells and benign breast epithelial cells. (a, b) TLK2 knockdown (KD) by esiRNA and siRNA transfection. (a) Cell proliferation was assessed by MTT assay in TLK2-high or low breast cancer cell lines or benign breast epithelial cells after TLK2 knockdown. Unt, untreated. Ctrl, control. Error bars represent the s.d. of three replicate measurements per condition. (b) Cell migration assessed by Boyden chamber transwell assay following TLK2 KD for $48 \mathrm{~h}$ in MCF7 and MDAMB361 cells using TLK2 esiRNA or siRNA. NIH 3 T3 cells are used as chemo-attractant for MCF7 and MDAMB361 cells. Error bars represent the s.d. of three replicates measurements per condition. (c) Clonogenic assay was performed following Dox-inducible shRNA silencing of TLK2 in breast cancer cells. $0.5 \mathrm{mg} \mathrm{ml}^{-1}$ of Dox was used for this assay. Dox, doxycycline. Error bars represent the s.d. of three replicate measurements per condition. (d) TLK2 inhibition suppresses anchorage-independent growth of MCF7 and MDAMB361 cells. Dox $\left(0.5 \mu \mathrm{g} \mathrm{ml}^{-1}\right)$ was administered for 2 days to induce TLK2 shRNA and then soft-agar colony formation assay was performed. Error bars represent the s.d. of three replicate measurements per condition. (e) The cell growth inhibition after TLK2 KD using a siRNA\#2 (with the same targeting sequence as the shTLK2) can be rescued by inducible TLK2 overexpression. Multiple silent mutations at the shTLK2 targeting region are introduced into the TLK2 ORF without affecting the amino acid sequence, to reduce the inhibition of ectopically expressed TLK2 by siRNA\#2. TLK2 expression was induced by treating MCF7 cells with 100 or $2,000 \mathrm{ng} \mathrm{ml}^{-1}$ Dox; siTLK2 was then transfected and incubated for 2 weeks for clonogenic assay. Error bars represent the s.d. of two replicate measurements per condition. $P$ values were calculated based on $t$-test. ${ }^{\star} P<0.05 ;{ }^{* \star} P<0.01 ;{ }^{{ }^{* \star \star}} P<0.001$. Dox, doxycycline.

mouse model, we transplanted the MCF7 cells inducibly expressing the TLK2 shRNA into female athymic nude mice, and assessed the potential therapeutic effect of TLK2 inhibition in the in vivo context (Fig. 5a). As most $\mathrm{ER}+$ breast tumours are treated with endocrine therapy, we also examined the combination effect of TLK2 inhibition together with tamoxifen, the most 

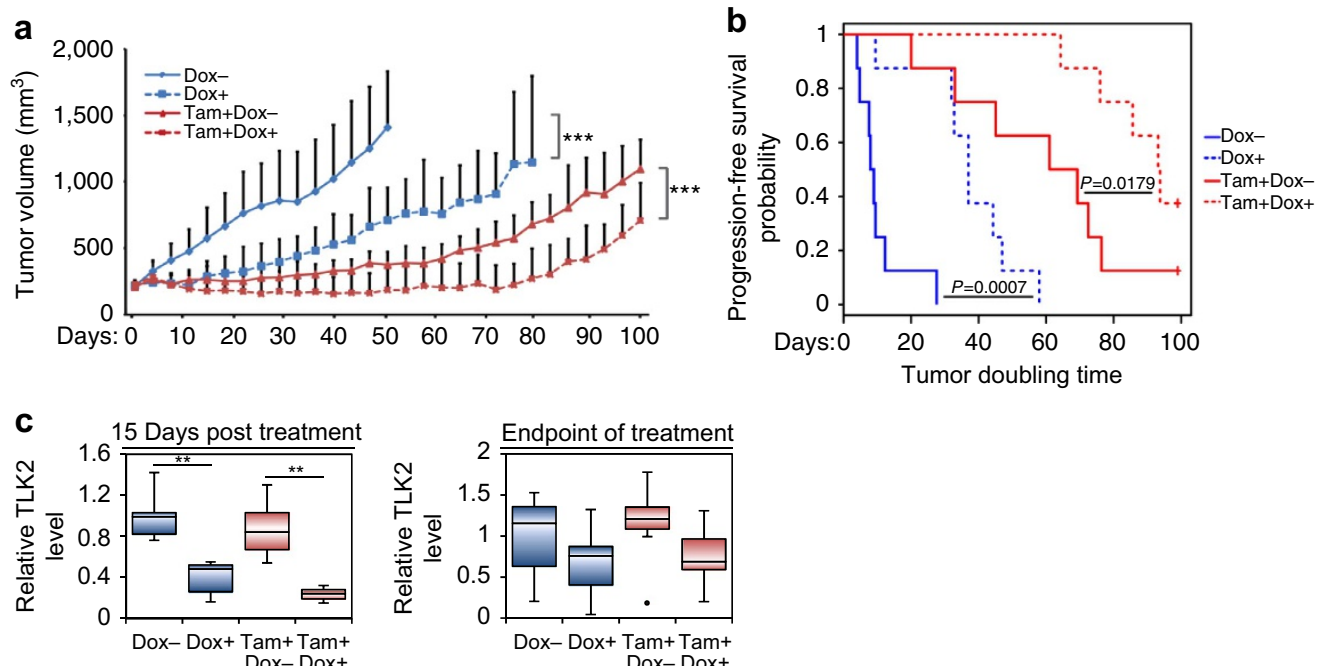

Figure 5 | The therapeutic effect of TLK2 inhibition in a MCF7 preclinical xenograft tumour model. (a) The effect of TLK2 inhibition in the MCF7 xenograft tumours inducibly expressing a TLK2 shRNA, in the presence or absence of concomitant tamoxifen treatment. The average tumour growth in each treatment group ( 8 mice per group). Error bars represent the s.d. of tumour volumes of 8 mice measurements per condition. $P$ values were calculated based on ANOVA to compare the tumour volumes. (b) Kaplan-Meier survival plot comparing the progression-free survival of different treatment groups (based on tumour-doubling time). Generalized Wilcoxon test was used to calculate the $P$ values for comparing progression-free survival between different treatment groups. (c) Quantitative western blot analysis of TLK2 protein expression in the tumours harvested after 15 days of treatment ( 5 mice/group), or at the end point ( 8 mice per group). Error bars represent the s.d. of relative TLK2 levels of 5 or 8 mice measurements per condition. ${ }^{\star} P<0.05 ;{ }^{\star \star} P<0.01$; ${ }^{\star \star \star} P<0.001$. Dox, doxycycline. Corresponding western blot images are shown in Supplementary Fig. 8.

commonly used endocrine agent. Upon tumour establishment, mice were randomized and treated with vehicle or tamoxifen (Tam), and further subdivided into \pm doxycycline treatments. To verify the effectiveness of TLK2 inhibition, a subset of tumours were harvested after 2 weeks of treatment, and analysed by western blot. To observe relative long-term therapeutic effects, the rest of the mice were monitored for up to 100 days depending on the duration of tumour control. Our result showed that TLK2 inhibition alone or in combination with tamoxifen substantially inhibited the growth of MCF7 xenograft tumours. Kaplan-Meier survival analysis revealed a significant increase in progression-free survival in TLK2 inhibition alone or concomitant TLK2 inhibition and tamoxifen treatment groups compared with the control groups (Fig. 5b). While the tumours in the combined treatment group still re-grew after 70 days of treatment, this could be attributable to the loss of TLK2 inhibition as suggested by the western blot analysis of tumours harvested at the end of the treatments (Fig. 5c; Supplementary Fig. 8). Such loss of target inhibition in the inducible knockdown tumour model after long-term induction of shRNA expression may be due to the selective pressure imposed by the target inhibition, which has been observed by others as well ${ }^{33}$. Together, these data provided a proof of concept for the therapeutic value of TLK2 inhibition in TLK2-amplified breast cancers.

The signalling changes following TLK2 inhibition. To systematically profile the cell signalling changes after TLK2 inhibition, we performed reverse phase protein array (RPPA) analysis of the MCF7 cells treated with TLK2 siRNAs as well as the xenograft tumours harvested after two weeks of TLK2 inhibition, using 200 validated antibodies against an array of key signalling molecules in cancer. We then isolated the consistently altered signalling molecules across different in vitro and in vivo TLK2 knockdown models based on student's $t$-test at $90 \%$ confidence (Fig. 6a; Supplementary Fig. 9). This revealed several consistently altered signalling molecules after TLK2 inhibition, among which BCL2 and $\mathrm{ER} \alpha$ are the most significantly down-regulated proteins (based on $t$-test, the average $P$ value for BCL2 is 0.0005 , and average $P$ value for ER $\alpha$ is 0.0252 ) (Fig. 6a, b). The downregulation of BCL2 and ER $\alpha$ after TLK2 knockdown were further verified by western blots (Fig. 6c). To test if these effects may be due to the off-target effects of TLK2 siRNAs, we examined the expression of BCL2 and ER $\alpha$ transcripts following TLK2 knockdown by quantitative PCR, which revealed no significant change or even upregulation of the mRNA levels of BCL2 and ER $\alpha$ (Fig. 6d). This suggests that the modulations of BCL2 and ER $\alpha$ by TLK2 knockdown are mainly at the protein level. To examine if TLK2 overexpression upregulates BCL2 and $\mathrm{ER} \alpha$ protein level in breast tumour tissues, we compared the BCL2 and ER $\alpha$ protein level in ER-positive breast cancers with or without TLK2 overexpression using the RPPA data available from TCGA (Supplementary Fig. 10). As a result, we found that ER $\alpha$ protein is significantly elevated in TLK2-high breast tumours (based on $t$-test, $P=0.041$ ), in spite of a slight increase of ESR1 transcript (based on $t$-test, $P=0.158$ ). While both BCL2 transcript and protein are slightly higher in TLK2-high tumours, such differences are not statistically significant. This suggests that TLK2 may play a role in modulating ER $\alpha$ protein level in breast tumours, whereas the BCL2 response may be an effect specific to TLK2 inhibition.

TLK2 silencing impedes G1/S transition and induces apoptosis. To examine the impact of TLK2 inhibition on cell cycle progression, we performed flow cytometry of DNA content after TLK2 knockdown in asynchronized MCF7 and MDAMB361 cells. As shown in Fig. 7a, TLK2 knockdown led to substantial increase of G1 phase cells and decrease of S-phase cells, suggesting delayed cell cycle progression through the G1/S border. TLK2 inhibition also potently induced apoptosis in MCF7 and MDAMB361 cells, as shown by Annexin V assay (Fig. 7b). To observe the dynamic cell cycle progression after TLK2 knockdown, we performed a series of flow cytometry analyses 

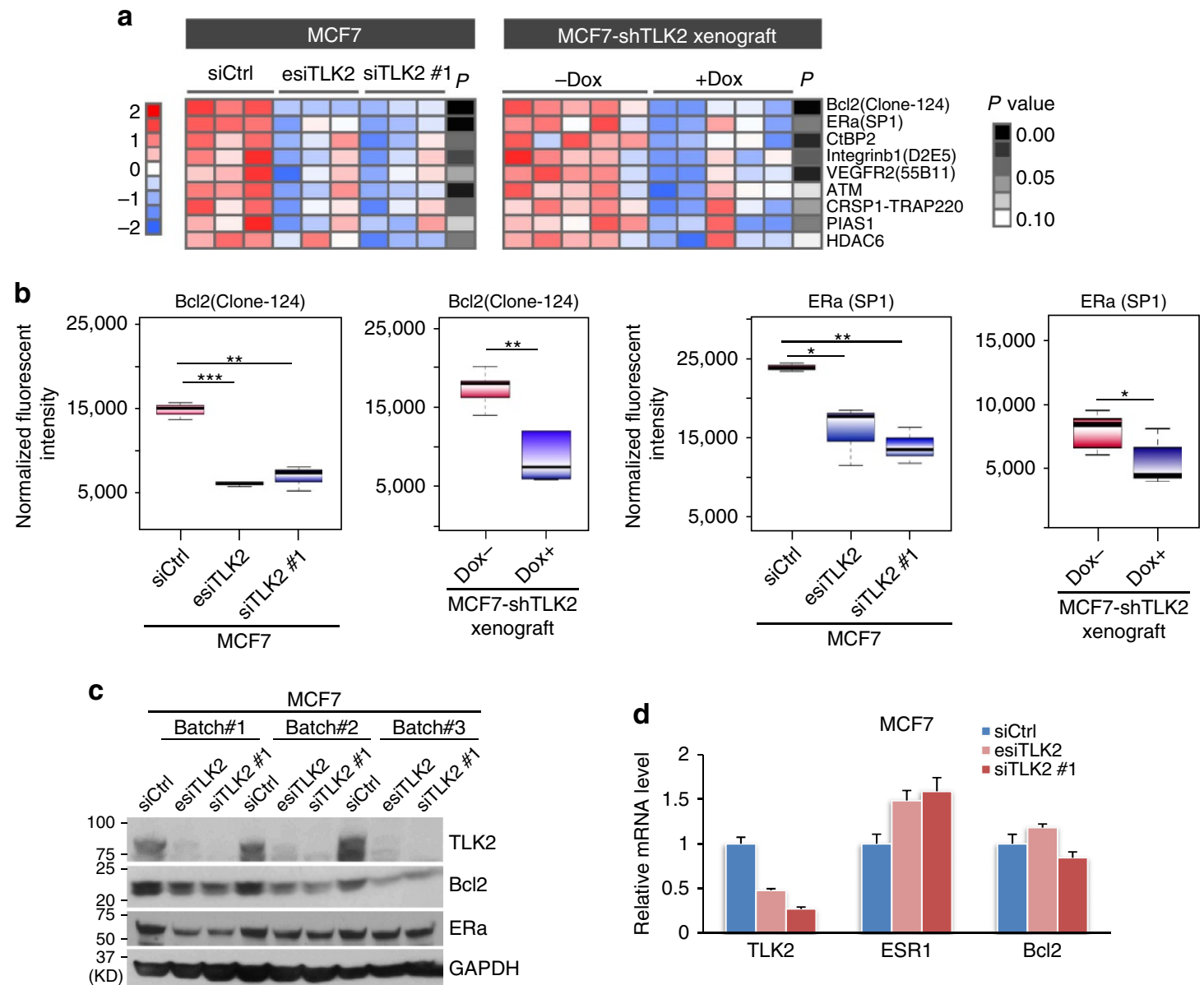

Figure 6 | RPPA profiling results after TLK2 knockdown in MCF7 cells or MCF7 xenograft tumours inducibly expressing shTLK2. (a) The heat-map of consistently altered signalling proteins revealed by RPPA analyses of MCF7 cells \pm TLK2 KD and MCF7 xenograft tumours harvested 2 weeks after induction of TLK2 inhibition. Proteins that showed a consistent trend of changes $(P<0.1)$ in both in vitro and in vivo models are shown in the heat-map, and are sorted by the mean $P$ values of different comparisons. For RPPA profiling, each knockdown experiment was repeated three times biologically. $P$ values were calculated based on $t$-test and are shown in grey scale. (b) Boxplots of normalized fluorescent intensities of Bcl2 or ER $\alpha$ after TLK2 knockdown in MCF7 cells (3 repeats for each group) or MCF7 xenograft tumours (5 tumours in each group). The whiskers indicate the max and min values and horizontal lines represent the 1st, 2 nd and 3rd quartiles. (c) Western blot validation of Bcl2 and ER $\alpha$ protein changes after TLK2 knockdown in MCF7 cells. (d) Q-PCR results quantifying relative mRNA level of TLK2, ER $\alpha$, or Bcl2 after TLK2 silencing by transfecting MCF7 cells with $10 \mathrm{nM}$ of siCtrl, esiTLK2, or siTLK2 \#1. Error bars represent the s.d. of three replicate measurements per condition.

following TLK2 knockdown by esiRNA in the MCF7 cells synchronized with a double thymidine (DT) block (Fig. 7c). Consistently, we observed delayed cell cycle progression through the G1/S border. In addition, western blot analysis revealed sustained high cyclin E level and low cyclin A level in response to TLK2 inhibition after cell cycle release from the DT block (Fig. 7d), suggesting that these cells were hindered from progressing into S-phase (Fig. 7e).

In addition, we also observed a markedly increased $\mathrm{p} 27$ protein level, and a decreased level of SKP2, the key E3-ligase of p27 (Fig. $7 \mathrm{~d})^{34}$. p27 inhibits the cyclin D/Cdk4 and cyclin E/Cdk2 complexes, and blocks cell cycle progression through the $\mathrm{G} 1 / \mathrm{S}$ border ${ }^{35}$. Thus the impeded G1/S transition may be attributable to increased p27 level. Interestingly, enhanced phosphorylation of p27 at T187 was also observed with TLK2 silencing. The phosphorylation of T187 is known to target p27 to the SCF ${ }^{\text {Skp2 }}$ ubiquitin ligase complex and proteasome-mediated degradation ${ }^{36}$. This suggests that the increased p27 protein level may be attributable to the decrease in SKP2, the key E3-ligase of p27, instead of impaired T187 phosphorylation. Furthermore, we also observed a decrease in phosphorylated Rb (S807/S811) after
TLK2 inhibition. Since p27 inhibits cyclin D/Cdk4 and cyclin $\mathrm{E} / \mathrm{Cdk} 2$, the key upstream kinases of Rb (ref. 37), the increased p27 after TLK2 knockdown may prevent Rb phosphorylation and subsequent E2F release ${ }^{38}$.

To verify the above results, we synchronized the MCF7 cells at $M$ phase via a nocodazole block, and the cell cycle was released in the condition of TLK2 inhibition by the siRNA\#1 that targets a different region from TLK2 esiRNA (Supplementary Fig. 4b). Cells were then subjected to flow cytometry analyses (Supplementary Fig. 11). The nocodazole mediated mitotic block allowed observing the cell cycle progression from $M$ to $G 1$, then to S-phase. Consistent with the DT block results, TLK2 inhibition delayed cell cycle progression through the G1/S border after MCF7 cells were released from mitotic arrest.

Differential cellular response to TLK2 or TLK1 inhibition. To observe the different roles of TLK2 and TLK1 in cell cycle regulation, we performed comparative cell cycle analyses of nocodazole-synchronized MCF7 cells in the condition of either TLK2 or TLK1 inhibition. TLK2 inhibition was accomplished 

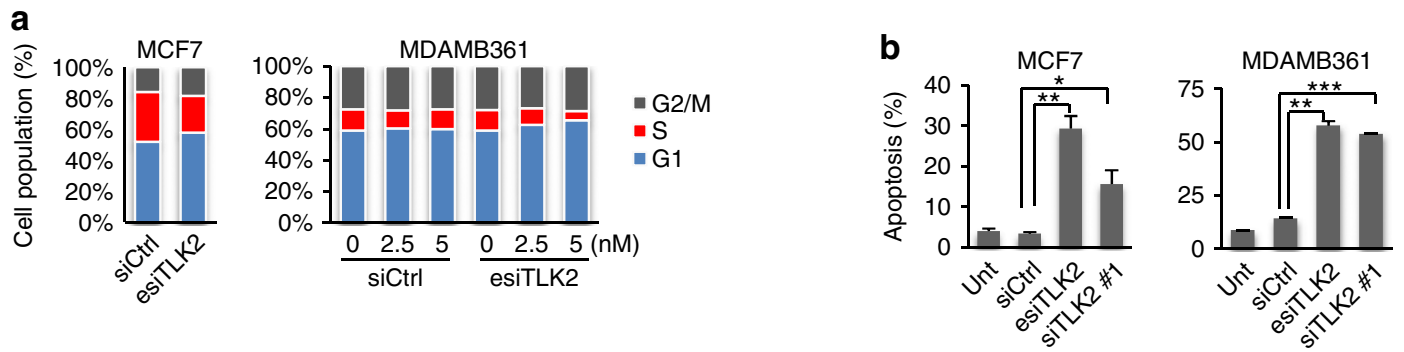

C

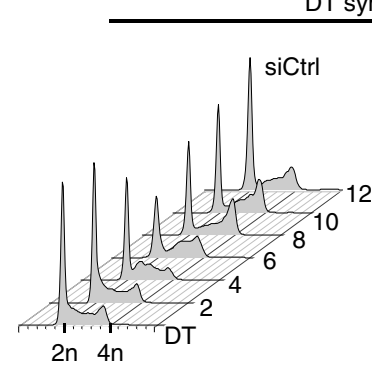

DNA Content

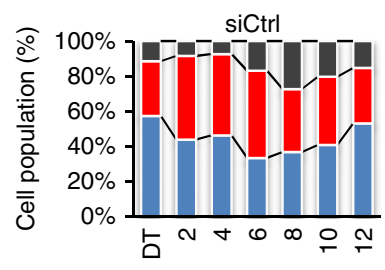

(h) After release
DT synchronized MCF7

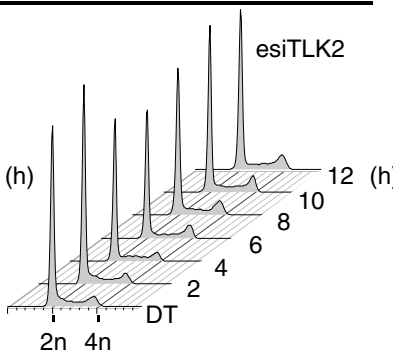

DNA Content

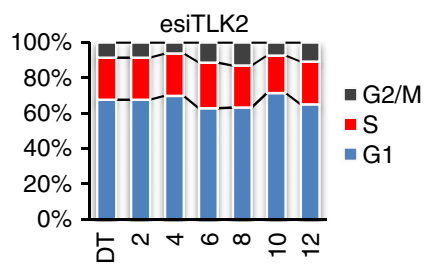

(h) After release

\section{d}

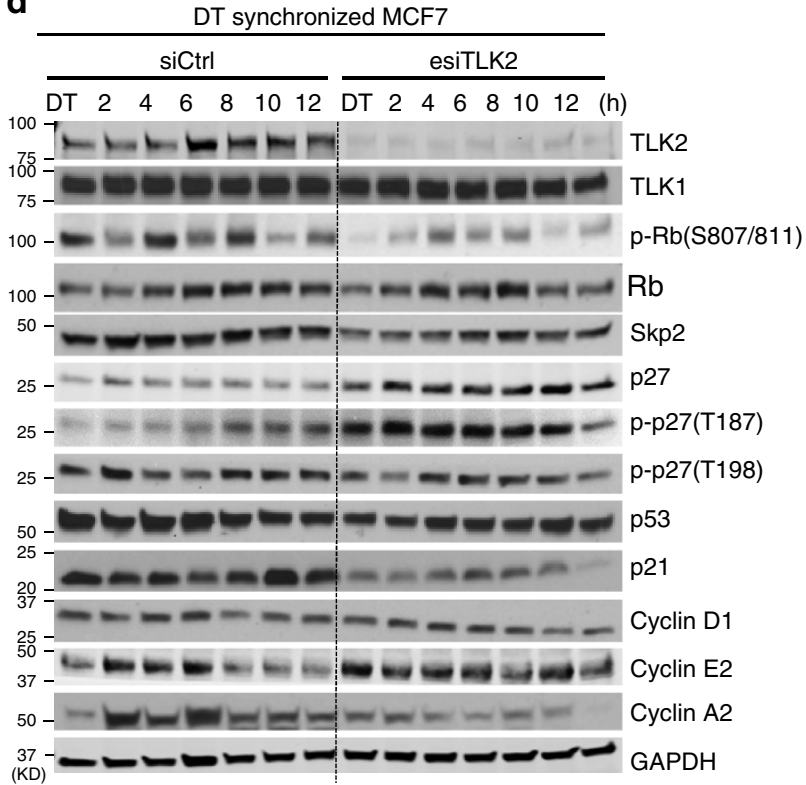

e

G1/S border
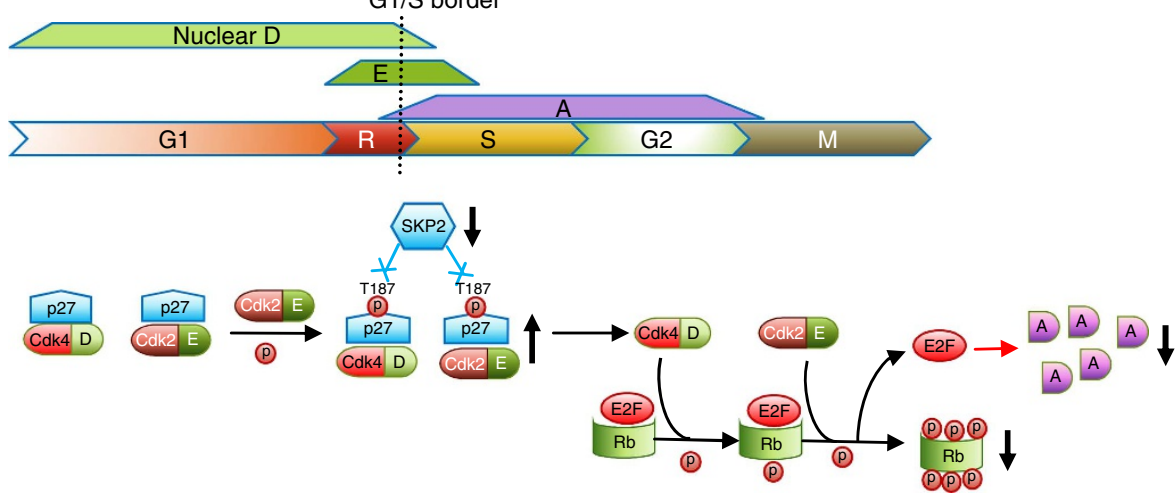

Figure 7 | TLK2 inhibition results in impaired G1/S progression and induction of apoptosis in TLK2-amplified luminal breast cancer cells. (a) Flow cytometry results showing cell cycle changes after TLK2 knockdown in asynchronized MCF7 and MDAMB361 cells. 10 nM siRNAs (for MCF7 cells) or indicated concentration of siRNAs (for MDAMB361 cells) were transfected for $72 \mathrm{~h}$. Ctrl, Control. (b) Cell apoptosis assessed by Annexin $V$ assay in asynchronized MCF7 and MDAMB361 cells following TLK2 knockdown via $20 \mathrm{nM}$ esiTLK2 or siTLK2\#1 transfection for $72 \mathrm{~h}$. Error bars represent the s.d. of two replicate measurements per condition. $P$ values are calculated based on $t$-test. ${ }^{\star} P<0.05 ;{ }^{* \star} P<0.01 ;{ }^{\star \star \star} P<0.001$. (c) Cell cycle profile of synchronized MCF7 cells (by double thymidine block) after TLK2 inhibition. After TLK2 knockdown for $24 \mathrm{~h}$, MCF7 cells were synchronized at the G1/S border using $2.5 \mathrm{mM}$ double thymidine (DT), and then released. Cells were collected every $2 \mathrm{~h}$ after cell cycle release for up to $12 \mathrm{~h}$, and analysed for DNA content using flow cytometry. (d) Western blot was done to examine the changes of key signalling molecules involved in G1/S cell cycle regulation using the cell lysates obtained from the same experiment as in 7c. 'DT' indicates synchronized MCF7 cells by DT block. (e) A schematic of normal G1/S cell cycle signalling and alternations following TLK2 inhibition (black arrows). In normal cell cycle, the cyclin E level starts to increase in late G1 phase, and then collapses as the cells enter $\mathrm{S}$ phase ${ }^{53}$, followed by increased cyclin A expression ${ }^{54,55}$. Rb regulates $\mathrm{G} 1 / \mathrm{S}$ transition by repressing the E2F transcription factors that control the expression of cyclin A. Once Rb is phosphorylated (that is, at S807/S811), it releases E2Fs, which will allow cells to enter S phase ${ }^{56,57}$. p27 inhibits the two G1 cyclin/cdk complexes, cyclin D/Cdk4 and cyclin E/Cdk2 (refs 36,40), both of which are the key upstream kinases of Rb (ref. 37). During normal G1/ $\mathrm{S}$ progression, the p27 proteins complexed with $\mathrm{G} 1$ cyclin/cdks were phosphorylated by the p27-free cyclin E/Cdk2 complexes at T187, which were then targeted for SKP2-mediated proteasome degradation ${ }^{58}$. D, Cyclin D. E, Cyclin E. A, Cyclin A. R, restriction point. 
using TLK2 esiRNA, whereas TLK1 was silenced using a specific siRNA previously documented ${ }^{39}$. To precisely determine the S-phase cell population, we incubated the cells with 5-bromo2'-deoxyuridine (BrdU), the S-phase DNA synthesis marker, before cell collection. Cells were then subjected to flow cytometry analysis, and cell cycle distribution was determined based on both DNA content and BrdU incorporation (Supplementary Fig. 12). In addition, cell signalling changes were monitored for up to $72 \mathrm{~h}$ to observe if there is enhanced apoptosis following impaired G1/S progression. Interestingly, while TLK2 inhibition led to delayed G1/S progression, TLK1 repression resulted in delayed S-phase progression, consistent with its known function in promoting chromatin assembly during S-phase (Fig. 8a) ${ }^{7}$. This suggests the distinct roles of TLK1 and TLK2 in cell cycle regulation.

Consistent with the previous data, western blots showed downregulation of SKP2, upregulation of p27, as well as increased cyclin $\mathrm{E}$ and decreased cyclin A levels after TLK2 inhibition (Fig. 8b). Further, we also observed repression of BCL2 and $\mathrm{ER} \alpha$ after TLK2 inhibition. BCL2 is an anti-apoptotic factor overexpressed in MCF7 cells ${ }^{40}$. Consistent with BCL2 repression, increased cleavage of caspase 3 and of PARP was observed in the TLK2-repressed MCF7 cells, suggesting induction of apoptosis. In contrast, inhibition of its paralog TLK1 did not significantly affect the BCL2 protein level, and there is no significant induction of cleaved caspase 3 or PARP (Fig. 8b, lower panel). To further verify this observation, we performed Annexin $\mathrm{V}$ assays after TLK2 or TLK1 silencing in MCF7 and MDAMB361 cells. While TLK2 inhibition significantly induced apoptosis, there was no significant increase in apoptosis after TLK1 silencing (Fig. 8c).

Taken together, these data suggest that, while TLK1 and TLK2 are close paralogs, these two kinases may play different roles in cell cycle regulation. Silencing of TLK1 or TLK2 appears to result in distinct cell cycle alterations and different effects on apoptosis in luminal breast cancer cells overexpressing TLK2.

\section{A kinase profiling data set reveals potential TLK2 inhibitors.} To identify potential TLK2 inhibitors, we investigated a publicly available kinase profiling data set that profiled the activity of 158 structurally diverse kinase inhibitors against 234 recombinant protein kinases ${ }^{41}$. We ranked these kinase inhibitors based on their activities against the TLK2 kinase, and evaluated their potential off-target effects based on the number of kinases against which the inhibitors presented stronger activities than TLK2 (Fig. 9a). Interestingly, two PKC inhibitors Go6983 and GF109203X were found to possess relatively strong and selective activities against TLK2. Both compounds are ATP-competitive inhibitors that bind to the ATP pocket of the PKC kinase catalytic domain. Kinase profiling data suggest that both Go6983 and GF109203X inhibit $\sim 98 \%$ of TLK2 kinase activity at $10 \mu \mathrm{M}$. We therefore performed in vitro kinase assays with myelin basic protein as a substrate, using recombinant active TLK2 proteins (SignalChem) treated with different doses of Go6983 or GF109203X (Fig. 9b). Both compounds resulted in potent inhibition of TLK2 activity at 5 or $10 \mu \mathrm{M}$. To assess their therapeutic effects via TLK2, we dosed MCF7 cells inducibly expressing TLK2 with $4 \mu \mathrm{M}$ Go6983 or GF109203X, and measured cell viability via clonogenic assays. Both compounds strongly inhibited cell viability, whereas induction of TLK2 overexpression can partially rescue the effect in a dose-dependent manner (Fig. 9c). This suggests that the therapeutic effects of these PKC inhibitors are at least partially via their actions against TLK2. Between these compounds, Go6983 showed a better inhibitory effect on cell viability and a stronger rescue effect from TLK2 overexpression. While Go6983 and GF109203X may not be applicable in vivo due to their off-target effects and the requirement of high doses to sufficiently inhibit TLK2 activity, these compounds could possibly serve as backbones for the future development of more potent and specific TLK2 inhibitors.

\section{Discussion}

In this study, ConSig-Amp analysis nominated TLK2 as a candidate kinase target upregulated by genomic amplifications in more aggressive form of luminal breast cancers. TLK2 amplification is independent of most known amplified oncogenes in breast cancer (that is, HER2, CCND1 and MYC), except RPSKB1 (Supplementary Fig. 3). While TLK2 is often co-amplified with RPSKB1 due to their vicinity (Supplementary Fig. 2), it is not uncommon that multiple closely located oncogenes are targeted by the same genomic amplifications in breast cancers, such as the co-amplifications of ERBB2 and GRB7 (ref. 42), FGFR1 and WHSC1L1 (ref. 43), or PAK1 and GAB2 (ref. 44). In fact, genomic amplifications in cancer usually affect multiple genes in the amplified regions. Besides luminal breast cancer cells, TLK2 is also overexpressed in a few ER-negative breast cancer cell lines (Fig. 2a). However, these cell lines typically do not harbour high TLK2 amplifications, and TCGA copy-number data suggest that TLK2 amplifications are much more frequent in ER-positive than negative breast cancers, 10.5\% versus $2.9 \%$ (Supplementary Fig. 1b). Consistently the latest phosphoproteomic study of TCGA breast tumours by The Clinical Proteomic Tumour Analysis Consortium (CPTAC) independently identified TLK2 as an amplicon-associated highly phosphorylated kinases in luminal breast cancer $^{11}$, which further support the significance of TLK2 amplification and its preferential association with luminal tumours. Our study is the first comprehensive analysis of TLK2 function in aggressive luminal breast cancers, which will timely complement the CPTAC paper. Our data showed that ectopic overexpression of TLK2 in the T47D luminal breast cancer cells markedly increased cell migration and invasion, whereas withdrawal of TLK2 expression eliminated this effect, suggesting the direct role of TLK2 in enhanced invasiveness. Furthermore, we found that TLK2 may involve the EGFR/SRC/FAK axis to enhance breast cancer cell invasiveness (Fig. $3 \mathrm{e}-\mathrm{g}$ ). Future studies will be needed to understand the precise mechanisms of TLK2-driven cell invasiveness and how exactly TLK2 interacts with the EGFR/SRC/FAK axis.

More important, breast cancer cells that harbour TLK2 amplifications appear to have been addicted to TLK2 overexpression, so that TLK2 knockdown causes potent growth inhibition and induction of apoptosis. In addition, we observed a selective effect of TLK2 inhibition on TLK2-high breast cancer cells versus TLK2-low breast cancer cells or benign breast epithelial cells. Of note, these effects appear to be sustained in the breast cancer cells that have already developed resistance to endocrine therapy. Our mechanistic studies suggest that TLK2 inhibition downregulates SKP2, upregulates p27, and impedes cell cycle progression through the G1/S border. In addition, we found that TLK2 inhibition consistently suppresses ER $\alpha$ and BCL2 protein level in vitro and in vivo, which may contribute to the substantially decreased cell proliferation and enhanced apoptosis after TLK2 inhibition. In contrast, inhibition of the TLK2 paralog, TLK1, resulted in a delay in S-phase progression, and did not significantly induce apoptosis (Fig. 8). This is the first observation on a role of TLK2 distinct from that of TLK1 in regulation of cell cycle progression-the latter (TLK1) has been known to promote chromatin assembly during S-phase.

It is interesting to note that ectopic expression of TLK2 in T47D cells did not increase cell proliferation (Fig. 3b). While TLK2 overexpression upregulates SKP2, the key E3-ubiquitin 
a

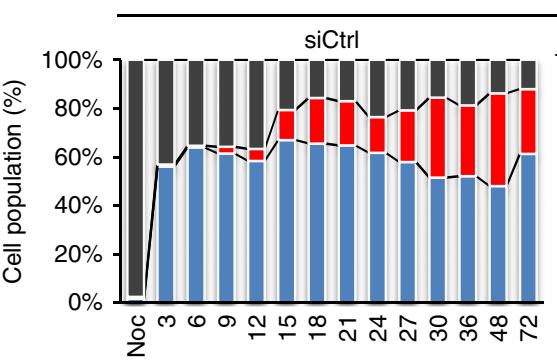

(h) After release
MCF7

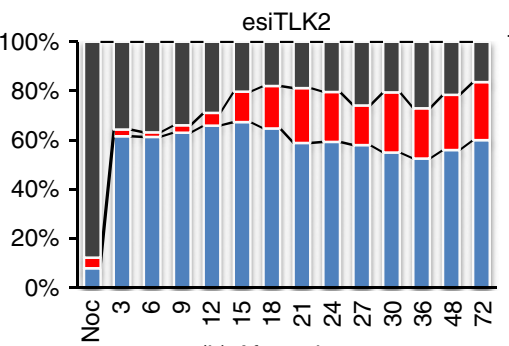

(h) After release

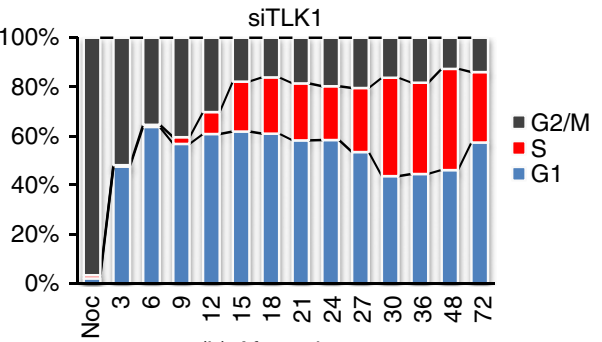

(h) After release

b

siCtrl

esiTLK2

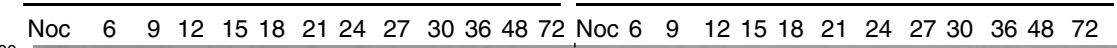

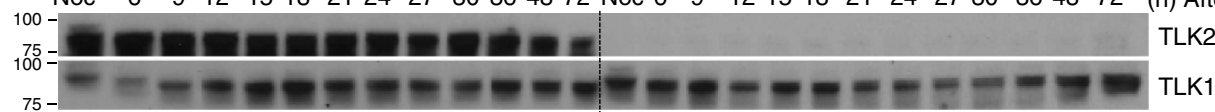

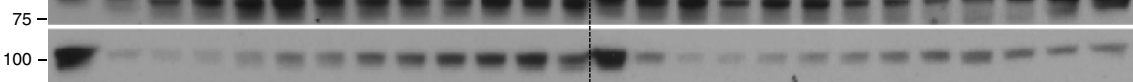

$\mathrm{p}-\mathrm{Rb}(\mathrm{S} 807 / 811)$

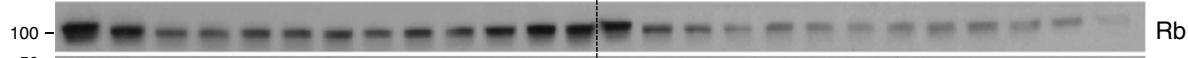

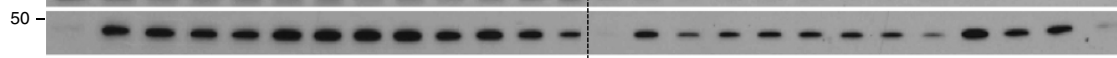

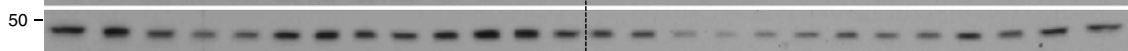

p-SKP2(S64)

SKP2

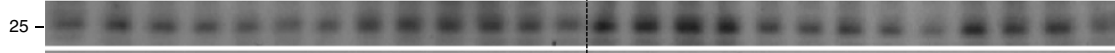

p-p27 (T187)

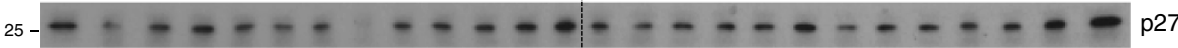

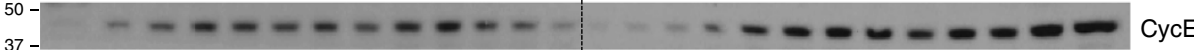

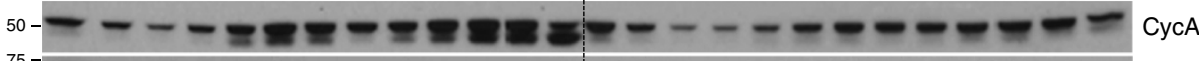

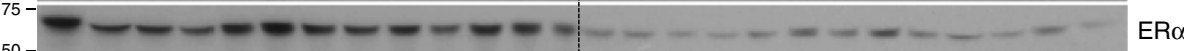

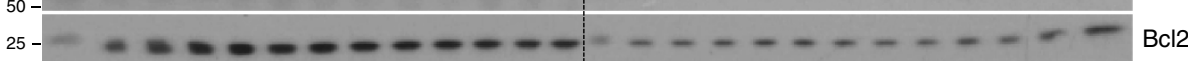

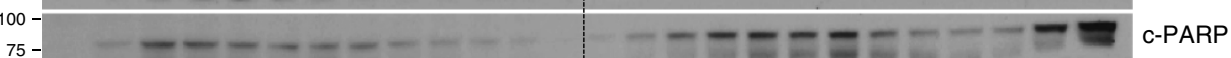

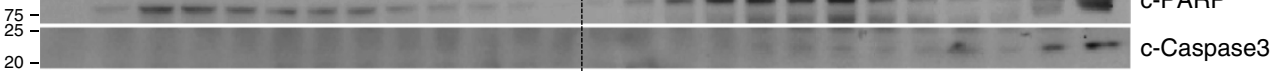

(KD)

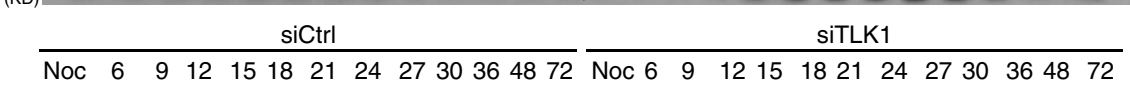
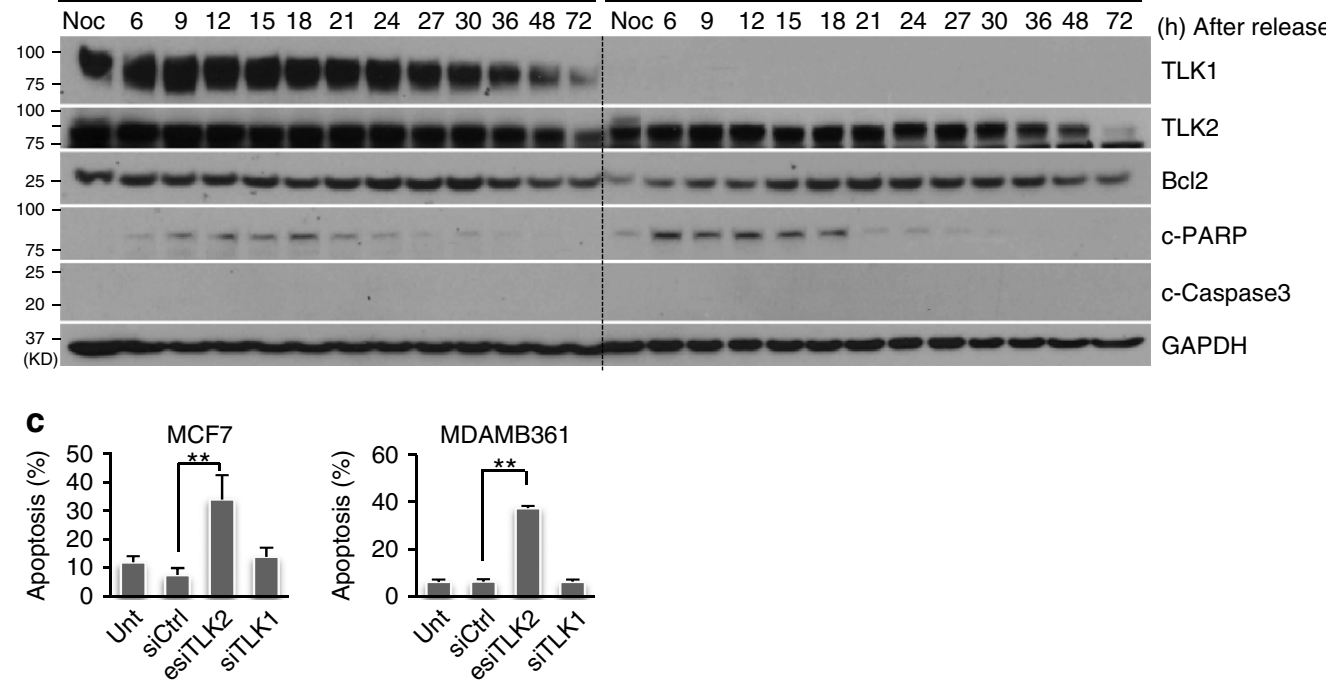

Figure 8 | TLK2-amplified luminal breast cancer cells respond differentially to TLK2 or TLK1 inhibition. (a) Cell cycle profile of MCF7 cells synchronized by nocodazole block after TLK2 or TLK1 knockdown. After TLK2 or TLK1 silencing by transfecting 10 nM of esiTLK2 or siTLK1 for 24 h, MCF7 cells were synchronized at mitosis using $200 \mathrm{nM}$ nocodazole for $15 \mathrm{~h}$, and then released. Cells were collected at the indicated time after cell cycle release. To precisely determine S-phase cell population, $10 \mu \mathrm{M}$ of BrdU was added for $1.5 \mathrm{~h}$ before cell collection. The cell cycle distributions were determined based on DNA content and BrdU incorporation (Supplementary Fig. 12). (b) Western blot was done to examine the changes of key signalling molecules involved in G1/S cell cycle regulation and apoptosis using the cell lysates obtained from same experiment as in Fig. 8a. 'Noc' indicates the MCF7 cells synchronized at mitosis by nocodazole block (before cell cycle release). (c) Cell apoptosis assessed by Annexin V assay in asynchronized MCF7 and MDAMB361 cells following $20 \mathrm{nM}$ of esiTLK2, siTLK1, or siCtrl treatment for $72 \mathrm{~h}$. Error bars represent the s.d. of two replicate measurements per condition.

$P$ values are calculated based on $t$-test. ${ }^{\star \star} P<0.01$. 

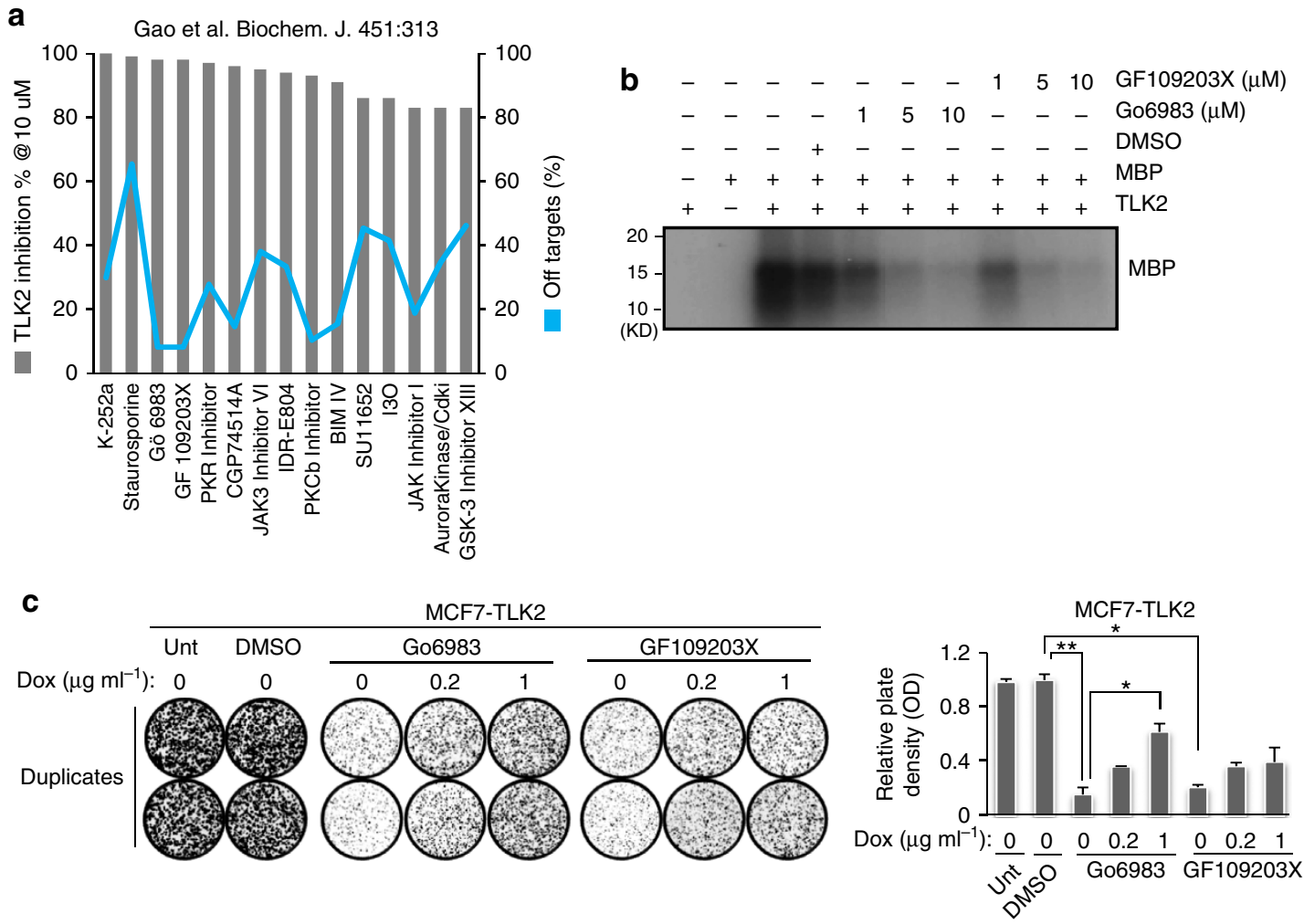

Figure 9 | Characterizing potential TLK2 inhibitors identified from a public kinase profiling data set. (a) Nominating potential TLK2 inhibitors based on analysis of a public kinase profiling data set ${ }^{41}$. Two potential TLK2 inhibitors were selected based on relatively strong TLK2 inhibition (shown in bar chart) and low number of off-targets (shown in line chart). (b) The activity of the potential TLK2 kinase inhibitors G06983 and GF109203X against TLK2 kinase were measured by in vitro kinase assay using recombinant active TLK2 protein. Myelin Basic Protein (MBP) was used as a substrate. (c) Clonogenic assays following Go6983 or GF109203X treatment of the MCF7 cells inducibly expressing TLK2. Go6983 (4 $\mu$ M) or GF109203X and 0.2 or $1 \mu \mathrm{g}$ ml ${ }^{-1}$ of Dox were administered. Error bars represent the s.d. of two replicate measurements per condition. $P$ values are calculated based on $t$-test. ${ }^{\star} P<0.05$;

${ }^{\star \star} P<0.01$.

ligase of p27 (ref. 45), we found that p27 protein level is not significantly down-regulated. This suggests that additional prerequisites may be needed to fulfill TLK2 function in modulating p27 and G1/S cell cycle progression. One possibility is that RPS6KB1 is frequently co-amplified with TLK2 (Supplementary Fig. 2), which may provide additional genetic background for the action of endogenously overexpressed TLK2 in breast cancer cells. Alternatively, the microenvironment of the primary tumours may provide additional signalling required for the action of endogenous TLK2. This reflects the limitations of ectopic expression cell line models in studying the action of amplified oncogenes, as such models may not possess the genetic background or microenvironment required for the full actions of endogenously amplified oncogenes, and therefore may not faithfully reproduce the mechanistic and phenotypic aspects of the oncogenes.

Furthermore, our in vivo data suggest that TLK2 inhibition may possess viable therapeutic value in TLK2-amplified luminal breast tumours. As shown by the study of a preclinical xenograft tumour model, TLK2 inhibition significantly improved progression-free survival. Nevertheless, we admit the limitations of our in vitro and in vivo cell line models on predicting therapeutic values, due to excessive clonal evolutions of in vitro cultured cells and lack of stromal interactions. Future studies will be required to further evaluate the therapeutic effect of TLK2 inhibition in patient-derived xenograft tumour models, and ultimately in clinical trials of breast cancer. Of note, TLK2 appears to be more frequently amplified than other known cell cycle kinases or checkpoint kinases ${ }^{46}$ in breast cancers as shown by TCGA copy-number data (Supplementary Table 3). The selective effect of TLK2 inhibition against TLK2-high breast cancer cells as compared with benign breast epithelial cells suggests a possible more selective cellular effect of TLK2 inhibitors as compared with the inhibitors of other cell cycle kinases that do not show cancer cell specificity. Moreover, we have identified two potential TLK2 inhibitors and tested their therapeutic activities against TLK2 in vitro. These compounds could serve as backbones for future drug development. Taken together, these facts position TLK2 as an attractive cell cycle kinase target for more aggressive luminal breast cancers that harbour TLK2 amplifications.

\section{Methods}

Integrative ConSig-amp analysis. To discover new therapeutic targets in ER + breast cancer, we analysed the copy number (Affymetrix SNP 6.0) and RNAseq (UNC RNAseqV2) data sets available for breast tumours from The Cancer Genome Atlas Project (TCGA) ${ }^{12}$. Normalized 'level 3' data (segmented by the CBS algorithm) (14) were directly applied in the analysis. First, the copy-number segments were matched with human genes based on physical coordinates to obtain gene-level copy-number data. The frequency of genomic amplification of each human gene in breast cancer was assessed; breast tumours with relative copy number at the respective gene locus more than 0.7 were considered as amplification positive. Genes that are amplified in $>5 \%$ of ER + tumours were nominated, and their expressions based on RNAseq data were correlated with copy-number data by Spearman's correlation statistics. The druggability of these genes was predicted based on a drug-target database compiled from multiple sources ${ }^{13-15}$. Then all candidates were ranked by the ConSig-amp score calculated by multiplying the Spearman's correlation coefficient by the concept signature (ConSig) score that we have developed that prioritizes functionally important genes underlying cancer by accessing their associations with cancer-related molecular concepts ${ }^{2}$. The ConSig 
scores are calculated using a cancer gene list $(n=385)$ compiled from the Cancer Gene Census (http://www.sanger.ac.uk/genetics/CGP/Census) and the Mitelman database (http://cgap.nci.nih.gov/Chromosomes/Mitelman), and a compiled molecular concept database including the $\mathrm{C} 1, \mathrm{C} 2, \mathrm{C} 3$ and $\mathrm{C} 5$ gene sets from MSigDb (http://www.broadinstitute.org/gsea/msigdb), and gene interactions from NCBI (ftp://ftp.ncbi.nlm.nih.gov/gene/GeneRIF) and Visant (http://visant.bu.edu/) databases. The detailed protocol to calculate the ConSig Score and the precomputed scores used in this study (for all human genes) are available in the website http://consig.cagenome.org (release 2). The top 50 druggable candidate oncogenes amplified in ER + breast cancers are provided in Supplementary Table 1 (ranked based on ConSig-amp score). The ConSig-amp scores range from 0 to 2.5. The ConSig-amp scores for ERBB2, PTK2, RPSKB1 and TLK2 are 2.49, $2.45,1.94$ and 1.55 respectively.

Gene expression data and survival analysis. To examine the prognostic value of TLK2 overexpression in ER-positive breast cancer, we analysed the overall survival data available for TCGA patients and correlated with the TLK2 gene expression data obtained from the level 3 RNAseq data. In addition, we also analysed the survival gene expression data sets by Loi et al. (GSE6532, Affymetrix U133 plus v2.0 $)^{20}$, and Molecular Taxonomy of Breast Cancer International Consortium (Metabric data set, Illumina HT-12 v3) ${ }^{18}$. Normalized gene expression data matrixes were used for survival analysis. To select optimal TLK2 probes for survival analysis, we aligned the TLK2 probes in the Illumina HT-12 v3 and Affymetrix U133 arrays with human reference genes. In the Illumina HT-12 v3 array, ILMN_1663486 is the only probe that specifically aligns to TLK2 but not to TLK2 homologues. In the Affymetrix U133 arrays, 212986_s_at and 212997_s_at are the only TLK2-specific probes; thus the mean of these two probes was used for subsequent survival analysis. All these TLK2-specific probes map to the last exon of TLK2. ILMN_1663486 is within the Affymetrix 212997_s_at probe region. Patients were divided into two groups (TLK2 high and the rest) based on the cutoff of median $+1 \times$ MAD (median absolute deviation). MAD is calculated using the $\mathrm{R}$ with default constant. Kaplan-Meier analyses were carried out using the $\mathrm{R}$ survival package. Follow-up time was constrained to a maximum of 10 years. $P$ values were calculated based on the log-rank test ( $P$ values were not adjusted for multiple comparisons). PAM50-based clinical subtypes of breast cancer for TCGA samples were derived from the UCSC Cancer Genome Browser (https://genome-cancer.ucsc.edu/) ${ }^{47,48}$. For the Affymetrix Human Exon 1.0 ST data for breast cancer cell lines ${ }^{21}$, exon expression signals were extracted using the RMA-sketch of Affymetrix power tools. TLK2 gene expression signals were summarized by taking the mean of the expression values of the probes mapping to the last exon of TLK2.

Cell culture. T47D, MDAMB361, CAMA1, ZR75-1, MCF10A and MCF12A cells were obtained from American Type Culture Collection (ATCC) included in the NCI-ATTC ICBP 45 cell line kit. 293FT cells used for lentivirus packaging were purchased from Invitrogen. MCF7 cells, a tamoxifen-resistant MCF7 clone (MCF7 TAM-R), and an oestrogen deprivation-resistant MCF7 clone (MCF ED-R) were obtained from Dr Rachel Schiff's lab ${ }^{32}$. MCF7 and T47D cells were cultured in RPMI 1640 (Cellgro) with 10\% fetal bovine serum (Thermo Fisher Scientific). MDAMB361 and 293FT cells were cultured in DMEM (Thermo Fisher Scientific) with $10 \%$ fetal bovine serum. MCF10A and MCF12A were cultured as described ${ }^{49}$. MCF7 Tam-R and ED-R cells were maintained in phenol red-free RPMI 1640 (Corning) containing 10\% charcoal-dextran treated fetal bovine serum (CD-FBS, Thermo Fisher Scientific), and to sustain the tamoxifen resistance, $10^{-7} \mathrm{M}$ tamoxifen was added to MCF7 TAM-R cells.

siRNA or esiRNA transfection. The TLK2-specific esiRNA (\#EHU113941), customized TLK2 siRNA\#2 (5'-CCCAGAAUAGUUAAGCUGU- $3^{\prime}$ ), TLK1 siRNA (\#SIHK2292), and control siRNAs (\#SIC001) were purchased from Sigma-Aldrich. In addition, customized TLK2 siRNA\#1 (5'-GAUAGAAAGACAACGGAAA- $3^{\prime}$ ), SMARTpool EGFR siRNA (E-003114-00-0005, \#1 5'-GUCUUAUCUAACUAUGAUG-3', \#2 $5^{\prime}$-UCACUCUCCAUAAAUGCUA-3' ${ }^{\prime}$, \#3 5'-GUAACAAGCUCACGCAGUU- $3^{\prime}$, \#4 $5^{\prime}$-GGAUAUUCUGAAAACCGUA- $\left.3^{\prime}\right)$, FAK ( $5^{\prime}$ AACCACCUGGGCCAGUAUUAUUU-3'), SRC siRNA (J-003175-16-0005, $5^{\prime}$ GGGAGAACCUCUAGGCACA-3') and control siRNA (D-001810-10-20) were purchased from Dharmacon. For transfection, 10-20 nM esiRNA or siRNA was applied using Lipofectamine RNAi MAX (Invitrogen) according to manufacturer's instructions.

MTT cell proliferation assay. Cells $(1,000-3,000)$ were seeded in 96-well plates $24 \mathrm{~h}$ before the siRNA or esiRNA transfection. Cell proliferation was analysed for 7 days by MTT assay using the Cell Proliferation kit I (Roche) following manufacturer's protocols.

Western blot. Cells were extracted in RIPA lysis buffer (Sigma-Aldrich), supplemented with complete protease inhibitor cocktail tablet (Roche). Protein samples were separated in SDS-PAGE gel and then transferred onto a $0.2 \mu \mathrm{m}$ nitrocellulose membrane. Primary antibodies were used with 1:500-1:2,000 dilution. The following antibodies were used for western blot: rabbit anti-TLK2 (Bethyl Laboratories, A301-257A, 1:1,000), rabbit anti-TLK1 (Bethyl Laboratories, A301-252A, 1:1,000), mouse anti-GAPDH (Santa Cruz, sc-32233, 1:2,000), rabbit anti-phospho p27 (T187) (Abcam, ab75908, 1:500), mouse anti-L1CAM (Abcam, ab3200, 1:1,000), rabbit anti-phospho p27 (T198) (Abcam, ab64949, 1:500), rabbit anti-p27 (Santa Cruz, sc-528, 1:500), rabbit anti-Cyclin A2 (Santa Cruz, H-432, 1:2,000), mouse anti-Bcl2 (Dako, M0887, 1:1,000). Rabbit anti-Cyclin D1 (\#2978, 1:1,000), rabbit anti-Cyclin E2 (\#4132, 1:1,000), rabbit anti-Skp2 (\#2652, 1:1,000), rabbit anti-phopho-p53 (S15) (\#9284, 1:1,000), rabbit anti-p53 (\#9282, 1:1,000), rabbit anti-p21 (\#2947, 1:1,000), rabbit anti-ER $\alpha$ (\#8644, 1:1,000), rabbit anti-EGFR (\#4267, 1:1,000), rabbit anti-phospho EGFR (Y845) (\#6963, 1:1,000), rabbit anti-phospho EGFR (Y1068) (\#3777, 1:1,000), rabbit anti-phopho FAK (Y397) (\#8556, 1:500), rabbit-anti FAK (\#13009, 1:1,000), rabbit anti-PAK1 (\#2602, 1:1,000), rabbit anti-HER2 (\#4290, 1:1,000), rabbit anti-phospho HER2 (Y1248) (\#2247, 1:1,000), rabbit anti-phospho HER2 (Y1221/1222) (\#2243, 1:1,000), rabbit anti-phospho HER2 (Y877) (\#2241, 1:1,000), rabbit anti-phospho Rb (S807/811) (\#8516, 1:1,000), mouse anti-Rb (\#9309, 1:1,000), rabbit anti-SRC (\#2123, 1:1,000), rabbit anti-phospho SRC (Y416) (\#6943, 1:1,000), rabbit anti-phospho AKT (S473) (\#4060, 1:1,000), rabbit anti-AKT (\#4691, 1:1,000), rabbit anti-phospho ERK1/2 (T202/Y204) (\#4370, 1:1,000), rabbit anti-ERK1/2 (\#4695, 1:1,000), rabbit anti-phospho p38 (T180/Y182) (\#4511, 1:1,000), rabbit anti-p38 (\#8690, 1:1,000), rabbit anti-phospho c-Jun (S63) (\#9261, 1:1,000), rabbit anti-c-myc (\#13987, 1:1,000), rabbit anti-c-Caspase 3 (\#9661, 1:500), and rabbit anti-c-PARP (\#5625, 1:1,000) were purchased from Cell Signalling. Uncropped western blots were shown in Supplementary Fig. 13.

Reverse-transcription PCR and quantitative PCR. Total RNA was isolated with the RNeasy Mini Kit (Qiagen) according to the manufacturer's protocol. Complementary DNA was synthesized from $1 \mu \mathrm{g}$ total RNA, using a Transcriptor First Strand cDNA Synthesis Kit (Roche) in the presence of both oligo (dT) and random primers. The sequences of all PCR primers are listed in Supplementary Table 2. The relative expression level of each target gene was determined using the comparative threshold cycle $(\mathrm{Ct})$ method and normalized to respective GAPDH controls.

Engineering Dox-inducible plasmids and stable cell lines. The full-length cDNA of TLK2 was purchased from Origene (Catalogue \#: SC115810), and the open reading frame (ORF) was subcloned into an inducible lentiviral pTINDLE vector provided by Dr Xuewen Pan. This vector contains an inducible promoter (pTRE-tight) and a transactivator (rtTA3) in a lentiviral backbone. We also engineered the ORF of Yellow Fluorescent Protein (YFP) into the pTINDLE vector as a control. TLK2 shRNA (5'-CCCAGAATAGTTAAGCTGT- $\left.3^{\prime}\right)$ and non-silencing controls ( $5^{\prime}$-ATCTCGCTTGGGCGAGAGTAAG-3') were purchased from Open Biosystems. The shRNA was engineered into another inducible lentiviral pINDUCER vector ${ }^{50}$. After lentivirus packaging, cells were infected by lentivirus containing doxycycline (Dox) inducible plasmid, adding $8 \mu \mathrm{g} \mathrm{ml}^{-1}$ polybrene. Stable cell lines expressing shTLK2 were established by sorting GFP-positive cells using a flow cytometric cell sorter, FACSAria (BD Biosciences). The stable lines expressing the TLK2 ORF were selected by treating with Geneticin (Invitrogen). $2 \mu \mathrm{g} \mathrm{ml}^{-1}$ of Dox (Sigma-Aldrich) was used for shTLK2 induction and $0,50,100$ or $200 \mathrm{ng} \mathrm{ml}^{-1}$ of Dox was used to express the TLK2 ORF. For inducible overexpression systems, TLK2 expression was induced for two weeks before the stable lines were subjected to phenotypic assays.

Clonogenic assay. Cells (3,000-5,000) were seeded in 6-well plates and incubated for 14-21 days. For the Dox-inducible TLK2 overexpression model, $100 \mathrm{ng} \mathrm{ml}^{-1}$ of Dox was added for 2 weeks before the clonogenic assay. For the Dox-inducible TLK2 knockdown model, $0.5 \mu \mathrm{g} \mathrm{ml}^{-1}$ of Dox was added for 2 days before the clonogenic assay. The colonies were stained with $0.5 \%$ crystal violet and $50 \%$ methanol and were counted by a GelCount colony counter (Oxford Optronix).

Soft-agar colony formation assay. Cells $(3,000-5,000)$ were suspended in growth medium containing $0.35 \%$ SeaPlaque Agarose (Lonza), and plated on $0.5 \%$ base agar in 6-well plates. Then cells were incubated at $37^{\circ} \mathrm{C}$ in $5 \% \mathrm{CO}_{2}$ for $14-21$ days, and colonies were counted using GelCount (Oxford Optronix Ltd.). For the Dox-inducible TLK2 overexpression model, $100 \mathrm{ng} \mathrm{ml}^{-1}$ of Dox was added for 2 weeks before the clonogenic assay. For the Dox-inducible TLK2 knockdown model, $0.5 \mu \mathrm{g} \mathrm{ml}^{-1}$ of Dox was added for 2 days before the clonogenic assay.

Transwell migration and invasion assay. Boyden chambers were used for transwell migration and invasion assays. Cells were serum-starved for $24 \mathrm{~h}$ and $5 \times 10^{4} \sim 3 \times 10^{5}$ cells were seeded with serum-free medium into the top of the transwell inserts with $8 \mu \mathrm{m}$ pore size for the migration assay, or into the top of the transwell coated with matrigel (BD Biosciences) for the invasion assay. In the bottom chamber, regular medium containing serum was added. To facilitate the migration of MCF7, MDAMB361, or T47D cells, NIH3T3 cells were seeded in 
the bottom chamber as a chemo-attractant. For the Dox-inducible TLK2 overexpression model, $0,50,100$, or $200 \mathrm{ng} \mathrm{ml}^{-1}$ of Dox was administered for 2 weeks before the migration and invasion assay. To verify the dependence of migration and invasion properties on TLK2 expression, Dox was withdrawn for 4 days following 2 weeks of Dox treatment to deplete the excess TLK2 protein. To observe the effect of SRC, EGFR or FAK inhibition on TLK2-driven cell motility, $20 \mathrm{nM}$ of siRNA targeting EGFR and FAK or $20-40 \mathrm{nM}$ of SRC siRNA (Dharmacon) were transfected for 3 days before perform the transwell migration assay. After $48-72 \mathrm{~h}$, the inserts were fixed in $4 \%$ formaldehyde and stained with hematoxylin and eosin. The migrated and invaded cells were counted by GelCount colony counter (Oxford Optronix Ltd.).

Immunoprecipitation assay. Rabbit monoclonal antibody against SRC (Cell Signalling \#2123) was conjugated with protein A/G-sepharose beads (Santa Cruz). $500 \mu \mathrm{g}$ of fresh protein lysates from cells were immunoprecipitated for overnight at $4^{\circ} \mathrm{C}$ with constant rotation. After washing three times with extraction buffer, proteins that co-immunoprecipitated were analysed by western blot as described previously. Protein lysate $(30-50 \mu \mathrm{g})$ was loaded as a control input.

Reverse phase protein array analysis. Reverse phase protein array assays were carried out as described previously with minor modifications ${ }^{51}$. Protein lysates were prepared from cultured cells or tissue samples with modified Tissue Protein Extraction Reagent (TPER) (Pierce) and a cocktail of protease and phosphatase inhibitors (Roche Life Science). The lysates were diluted into $0.5 \mathrm{mg} \mathrm{ml}^{-1}$ of total protein in SDS sample buffer and denatured on the same day. The Aushon 2470 Arrayer (Aushon BioSystems) with a 40 pin $(185 \mu \mathrm{m})$ configuration was used to spot samples and control lysates onto nitrocellulose-coated slides (Grace Bio-labs) using an array format of 960 lysates/slide (2880 spots/slide). The slides were processed as described ${ }^{51}$ and probed with a set of 200 antibodies against total and phosphoprotein proteins using an automated slide stainer Autolink 48 (Dako). Each slide was incubated with one specific primary antibody and negative control slide was incubated with antibody diluent instead of primary antibody. Primary antibody binding was detected using a biotinylated secondary antibody followed by streptavidin-conjugated IRDye680 fluorophore (LI-COR Biosciences). Total protein content of each spotted lysate was assessed by fluorescent staining with Sypro Ruby Protein Blot Stain according to the manufacturer's instructions (Molecular Probes). Flurosecent-labeled slides were scanned on a GenePix AL4200 scanner, and the images were analysed with GenePix Pro 7.0 (Molecular Devices). Total fluorescence signal intensities of each spot were obtained after subtraction of the local background signal for each slide and were then normalized for variation in total protein, background and non-specific labelling using a group-based normalization method as described ${ }^{51}$. For each spot on the array, the-backgroundsubtracted foreground signal intensity was subtracted by the corresponding signal intensity of the negative control slide (omission of primary antibody) and then normalized to the corresponding signal intensity of total protein for that spot. The median of the triplicate experimental values (normalized signal intensity) is taken for each sample for subsequent statistical analysis. $T$-tests are performed using Perl module 'Statistics::T-Test'

FACS analysis of cell cycle and apoptosis. For cell cycle analysis, cells were fixed in $70 \% \mathrm{EtOH}$ and then stained with propidium iodide (Sigma-Aldrich). For cell apoptosis analysis, cells were stained using the Annexin V-FITC apoptosis detection kit following manufacturer's protocols (Abcam). Cells were analysed using FACSCantoll cell analyzer (BD Biosciences) and Flowjo software.

Double thymidine or nocodazole block and BrdU incorporation. MCF7 cells were blocked at the G1/S border or in mitosis using the following protocols. MCF7 cells were blocked with $2.5 \mathrm{mM}$ Thy for $18 \mathrm{~h}$, released for $9 \mathrm{~h}$ after washing with PBS for three times, and then blocked again with $2.5 \mathrm{mM}$ Thy for $17 \mathrm{~h}$. To synchronize MCF7 cells in mitosis, cells were incubated with $200 \mathrm{nM}$ of nocodazole for $15 \mathrm{~h}$. After shaking off the cells, floating cells were collected to obtain the mitotic cell population and then cells were released by washing with PBS for three times. To precisely determine the S-phase cell population, $10 \mu \mathrm{M}$ of BrdU was added for $1.5 \mathrm{~h}$ before cell collection.

Fluorescence in situ hybridization assay. For FISH analysis, exponentially growing cells were treated with Colcemid $\left(0.04 \mu \mathrm{g} \mathrm{ml}^{-1}\right)$ for one hour at $37^{\circ} \mathrm{C}$ followed by hypotonic treatment $(0.075 \mathrm{M} \mathrm{KCl})$ for $20 \mathrm{~min}$ at room temperature. Cells were fixed in a methanol and acetic acid (3:1 by volume) mixture for $15 \mathrm{~min}$, and washed three times in the fixative. Slides were prepared by dropping the cell suspension on wet slides and air drying. FISH was performed on these slides using TLK2 (Red 5-Rox dUTP) and centromere 17 (Green 5-Fluorescein dUTR) probes from Empire Genomics, Buffalo, NY. Probe $(10 \mu \mathrm{l})$ was placed on each slide, covered with cover glass and sealed with rubber cement. The slides and the probe were co-denatured at $72^{\circ} \mathrm{C}$ for $3 \mathrm{~min}$ in ThermoBrite hybridzer, and then incubated at $37^{\circ} \mathrm{C}$ in a humid chamber overnight. The slides were washed in
$0.4 \mathrm{XSSC} / 0.3 \%$ Tween 20 at $72{ }^{\circ} \mathrm{C}$ for $2 \mathrm{~min}$ and in $2 \times \mathrm{SSC}$ at room temp for $2 \mathrm{~min}$. The slides were counterstained with DAPI, and the images were captured using Nikon 80i microscope equipped with a cooled-charge coupled devices (CCD) camera. A total of 50 interphase nuclei were analysed to determine the amplification status.

In vivo xenograft experiments. All animal experiments have been approved by the BCM Institutional Animal Care and Use Committee. MCF7 cells $\left(7.5 \times 10^{6}\right)$ with Dox-dependent expression of shTLK2 were injected bilaterally to 4-6 week old female athymic nude mice (Harlan Sprague-Dawley) supplemented with $17 \beta$-estradiol pellets. Xenograft tumours of the MCF7 models were successfully engrafted in 32 mice which were randomized into \pm doxycycline (Dox) with or without tamoxifen treatment ( 8 mice per group). Briefly, when tumours reached $200 \mathrm{~mm}^{3}$, tamoxifen $\left(25 \mu \mathrm{g} \mathrm{kg}^{-1}\right.$ body weight, 5 days weekly) was injected subcutaneously and $0.2 \mathrm{mg} \mathrm{ml}^{-1}$ Dox were administered with drinking water. The growth of the xenograft tumours was monitored twice per week and tumour volume was measured using the formula; tumour volume $=1 / 2\left(\right.$ length $\times$ width $^{2}$ ). Mice were sacrificed and tumours were harvested when they reached $1,500 \mathrm{~mm}^{3}$, or at the end of the experiment. To observe relative long-term therapeutic effects, mice were monitored for up to 100 days depending on the duration of tumour control. An additional 5 mice per group were included for the analyses of biomarkers, for which tumours were harvested after two weeks of treatments.

Statistical analysis. The results of the in vitro experiments were analysed by Student's $t$-tests, and all data are shown as mean \pm s.d.. For the in vivo study, the generalized Wilcoxon test was used for progression-free survival analysis and ANOVA was applied for tumour volume analysis in different treatment groups.

Data availability. The TCGA RNAseq and copy-number data sets used in this study are available from TCGA portal (http://cancergenome.nih.gov/). The Metabric copy-number and gene expression data sets are available from European GenomePhenome Archive (EGA: http://www.ebi.ac.uk/ega/), under accession number EGAS00000000083. Copy-number (Affymetrix SNP 6.0 array) and gene expression data (Affymetrix GeneChip Human Exon 1.0 ST Array) for breast cancer cell lines are available from EGA under accession number EGAS00000000059 and ArrayExpress (http://www.ebi.ac.uk/arrayexpress/) under accession number E-MTAB-181 respectively ${ }^{21}$. The RPPA data for the MCF7-TLK2 knockdown models are available in Supplementary Data 1. All other data is included in the Article or Supplementary Files or available from the authors upon request.

\section{References}

1. Guha, M. Blockbuster dreams for Pfizer's CDK inhibitor. Nat. Biotechnol. 31, 187 (2013)

2. Wang, X. S. et al. An integrative approach to reveal driver gene fusions from paired-end sequencing data in cancer. Nat. Biotechnol. 27, 1005-1011 (2009).

3. Wang, X.-S. et al. Characterization of KRAS rearrangements in metastatic prostate cancer. Cancer Discov. 1, 35-43 (2011).

4. Veeraraghavan, J. et al. Recurrent ESR1-CCDC170 rearrangements in an aggressive subset of oestrogen receptor-positive breast cancers. Nat. Commun 5, 4577 (2014).

5. Fan, Y. et al. Amplification and over-expression of MAP3K3 gene in human breast cancer promotes formation and survival of breast cancer cells. J. Pathol. 232, 75-86 (2014).

6. Sillje, H. H. \& Nigg, E. A. Identification of human Asf1 chromatin assembly factors as substrates of Tousled-like kinases. Curr. Biol. 11, 1068-1073 (2001).

7. Labussiere, H., Hayette, S., Tigaud, I., Michallet, M. \& Nicolini, F. E. Treatment of chronic myeloid leukemia in 2007. Bull. Cancer 94, 863-869 (2007).

8. Han, Z., Riefler, G. M., Saam, J. R., Mango, S. E. \& Schumacher, J. M. The C. elegans Tousled-like kinase contributes to chromosome segregation as a substrate and regulator of the Aurora B kinase. Curr. Biol. 15, 894-904 (2005).

9. Sillje, H. H., Takahashi, K., Tanaka, K., Van Houwe, G. \& Nigg, E. A. Mammalian homologues of the plant Tousled gene code for cell-cycle-regulated kinases with maximal activities linked to ongoing DNA replication. EMBO J. 18, 5691-5702 (1999).

10. Kelemen, L. E. et al. Genetic variation in the chromosome $17 \mathrm{q} 23$ amplicon and breast cancer risk. Cancer Epidemiol. Biomarkers Prev. 18, 1864-1868 (2009).

11. Philipp Mertins et al. Proteogenomics connects somatic mutations to signalling in breast cancer. Nature 534, 55-62 (2016).

12. Cancer Genome Atlas Network. Comprehensive molecular portraits of human breast tumours. Nature 490, 61-70 (2012).

13. Wishart, D. S. et al. DrugBank: a comprehensive resource for in silico drug discovery and exploration. Nucleic Acids Res. 34, D668-D672 (2006). 
14. Chen, X., Ji, Z. L. \& Chen, Y. Z. TTD: therapeutic target database. Nucleic Acids Res. 30, 412-415 (2002).

15. Anastassiadis, T., Deacon, S. W., Devarajan, K., Ma, H. \& Peterson, J. R. Comprehensive assay of kinase catalytic activity reveals features of kinase inhibitor selectivity. Nat. Biotechnol. 29, 1039-1045 (2011).

16. Santarius, T., Shipley, J., Brewer, D., Stratton, M. R. \& Cooper, C. S. A census of amplified and overexpressed human cancer genes. Nat. Rev. Cancer 10, 59-64 (2010).

17. Glenisson, M. et al. Identification of new candidate therapeutic target genes in triple-negative breast cancer. Genes Cancer 3, 63-70 (2012).

18. Curtis, C. et al. The genomic and transcriptomic architecture of 2,000 breast tumours reveals novel subgroups. Nature 486, 346-352 (2012).

19. Parker, J. S. et al. Supervised risk predictor of breast cancer based on intrinsic subtypes. J. Clin. Oncol. 27, 1160-1167 (2009).

20. Loi, S. et al. Definition of clinically distinct molecular subtypes in estrogen receptor-positive breast carcinomas through genomic grade. J. Clin. Oncol. 25, 1239-1246 (2007)

21. Heiser, L. M. et al. Subtype and pathway specific responses to anticancer compounds in breast cancer. Proc. Natl Acad. Sci. USA 109, 2724-2729 (2012).

22. Zhang, S. et al. SRC family kinases as novel therapeutic targets to treat breast cancer brain metastases. Cancer Res. 73, 5764-5774 (2013).

23. Mayer, E. L. \& Krop, I. E. Advances in targeting SRC in the treatment of breast cancer and other solid malignancies. Clin. Cancer Res. 16, 3526-3532 (2010).

24. Tai, Y. L., Chen, L. C. \& Shen, T. L. Emerging roles of focal adhesion kinase in cancer. Biomed. Res. Int. 2015, 690690 (2015).

25. Bolos, V., Gasent, J. M., Lopez-Tarruella, S. \& Grande, E. The dual kinase complex FAK-Src as a promising therapeutic target in cancer. Onco Targets Ther. 3, 83-97 (2010).

26. Chung, I. et al. Spatial control of EGF receptor activation by reversible dimerization on living cells. Nature 464, 783-787 (2010).

27. Duxbury, M. S. et al. RNA interference targeting focal adhesion kinase enhances pancreatic adenocarcinoma gemcitabine chemosensitivity. Biochem. Biophys. Res. Commun. 311, 786-792 (2003).

28. Chu, I. et al. p27 phosphorylation by Src regulates inhibition of cyclin E-Cdk2. Cell 128, 281-294 (2007).

29. Lu, G. et al. Phosphorylation of ETS1 by Src family kinases prevents its recognition by the COP1 tumor suppressor. Cancer Cell 26, 222-234 (2014)

30. Majid, S. et al. miR-23b represses proto-oncogene Src kinase and functions as methylation-silenced tumor suppressor with diagnostic and prognostic significance in prostate cancer. Cancer Res. 72, 6435-6446 (2012).

31. Theis, M. \& Buchholz, F. MISSION esiRNA for RNAi screening in mammalian cells. J. Vis. Exp. 39, e2008 (2010).

32. Morrison, G. et al. Therapeutic potential of the dual EGFR/HER2 inhibitor AZD8931 in circumventing endocrine resistance. Breast Cancer Res. Treat. 144, 263-272 (2014).

33. Cheng, H., Snoek, R., Ghaidi, F., Cox, M. E. \& Rennie, P. S. Short hairpin RNA knockdown of the androgen receptor attenuates ligand-independent activation and delays tumor progression. Cancer Res. 66, 10613-10620 (2006).

34. Kossatz, U. et al. Skp2-dependent degradation of p27kip1 is essential for cell cycle progression. Genes Dev. 18, 2602-2607 (2004).

35. Toyoshima, H. \& Hunter, T. p27, a novel inhibitor of G1 cyclin-Cdk protein kinase activity, is related to p21. Cell 78, 67-74 (1994).

36. Ganoth, D. et al. The cell-cycle regulatory protein Cks1 is required for SCF(Skp2)-mediated ubiquitinylation of p27. Nat. Cell Biol. 3, 321-324 (2001).

37. Zarkowska, T. \& Mittnacht, S. Differential phosphorylation of the retinoblastoma protein by G1/S cyclin-dependent kinases. J. Biol. Chem. 272, 12738-12746 (1997)

38. Harbour, J. W., Luo, R. X., Dei Santi, A., Postigo, A. A. \& Dean, D. C. Cdk phosphorylation triggers sequential intramolecular interactions that progressively block Rb functions as cells move through G1. Cell 98, 859-869 (1999).

39. Takayama, Y. et al. Silencing of Tousled-like kinase 1 sensitizes cholangiocarcinoma cells to cisplatin-induced apoptosis. Cancer Lett. 296, 27-34 (2010).

40. Akar, U. et al. Silencing of Bcl-2 expression by small interfering RNA induces autophagic cell death in MCF-7 breast cancer cells. Autophagy 4, 669-679 (2008).

41. Gao, Y. et al. A broad activity screen in support of a chemogenomic map for kinase signalling research and drug discovery. Biochem. J. 451, 313-328 (2013).

42. Pradip, D., Bouzyk, M., Dey, N. \& Leyland-Jones, B. Dissecting GRB7-mediated signals for proliferation and migration in HER2 overexpressing breast tumor cells: GTP-ase rules. Am. J. Cancer Res. 3 , 173-195 (2013)
43. Yang, Z. Q., Liu, G., Bollig-Fischer, A., Giroux, C. N. \& Ethier, S. P. Transforming properties of 8p11-12 amplified genes in human breast cancer. Cancer Res. 70, 8487-8497 (2010).

44. Bentires-Alj, M. et al. A role for the scaffolding adapter GAB2 in breast cancer. Nat. Med. 12, 114-121 (2006).

45. Tsvetkov, L. M., Yeh, K. H., Lee, S. J., Sun, H. \& Zhang, H. p27(Kip1) ubiquitination and degradation is regulated by the SCF(Skp2) complex through phosphorylated Thr187 in p27. Curr. Biol. 9, 661-664 (1999).

46. Lapenna, S. \& Giordano, A. Cell cycle kinases as therapeutic targets for cancer Nat. Rev. Drug Discov. 8, 547-566 (2009).

47. Goldman, M. et al. The UCSC Cancer Genomics Browser: update 2013. Nucleic Acids Res. 41, D949-D954 (2013).

48. Koboldt, D. C. et al. Comprehensive molecular portraits of human breast tumours. Nature 490, 61-70 (2012).

49. Debnath, J., Muthuswamy, S. K. \& Brugge, J. S. Morphogenesis and oncogenesis of MCF-10A mammary epithelial acini grown in three-dimensional basement membrane cultures. Methods 30, 256-268 (2003).

50. Meerbrey, K. L. et al. The pINDUCER lentiviral toolkit for inducible RNA interference in vitro and in vivo. Proc. Natl Acad. Sci. USA 108, 3665-3670 (2011).

51. Chang, C. H. et al. Mammary stem cells and tumor-initiating cells are more resistant to apoptosis and exhibit increased DNA repair activity in response to DNA damage. Stem Cell Rep. 5, 378-391 (2015).

52. Itamochi, H. et al. Checkpoint kinase inhibitor AZD7762 overcomes cisplatin resistance in clear cell carcinoma of the ovary. Int. J. Gynecol. Cancer 24, 61-69 (2014).

53. Aleem, E., Kiyokawa, H. \& Kaldis, P. Cdc2-cyclin E complexes regulate the G1/S phase transition. Nat. Cell Biol. 7, 831-836 (2005).

54. Chibazakura, T. et al. Cyclin A promotes S-phase entry via interaction with the replication licensing factor Mcm7. Mol. Cell Biol. 31, 248-255 (2011).

55. Yam, C. H., Fung, T. K. \& Poon, R. Y. Cyclin A in cell cycle control and cancer Cell Mol. Life Sci. 59, 1317-1326 (2002).

56. Knudsen, E. S. \& Wang, J. Y. Differential regulation of retinoblastoma protein function by specific Cdk phosphorylation sites. J. Biol. Chem. 271, 8313-8320 (1996).

57. Knudsen, E. S. \& Wang, J. Y. Dual mechanisms for the inhibition of E2F binding to RB by cyclin-dependent kinase-mediated RB phosphorylation. Mol. Cell Biol. 17, 5771-5783 (1997).

58. Xu, X., Nakano, T., Wick, S., Dubay, M. \& Brizuela, L. Mechanism of Cdk2/Cyclin E inhibition by p27 and p27 phosphorylation. Biochemistry 38, 8713-8722 (1999).

\section{Acknowledgements}

This study was supported by CDMRP grants W81XWH-12-1-0166 (X.-S.W.), W81XWH-12-1-0167 (R.S.), W81XWH-13-1-0431 (J.V.), W81XWH-13-1-0201 (X.C.), Susan G. Komen foundation PDF12231561 (J.A.K.), PDF15333523 (X.W.), and PG12221410 (X.-S.W., R.S.), NIH grants R01-CA181368 (X.-S.W.), R01-CA183976 (X.-S.W.), and P30-125123-06, Nancy Owens Memorial Foundation (X.-S.W.), Commonwealth of PA Tobacco Phase 15 Formula Fund (X.-S.W.), Stand Up 2 Cancer Dream Team Translational Research Grant (grant Number SU2C-AACR-DT0409) (R.S.), and the Breast Cancer Research Foundation (BCRF) (R.S.). The results published here are in part based upon data generated by TCGA (dbGaP accession: phs000178.v6.p6). This study also makes use of data generated by the Molecular Taxonomy of Breast Cancer International Consortium (funding for that project was provided by Cancer Research UK and the British Columbia Cancer Agency Branch). The computational infrastructure was supported by the BCM Dan L. Duncan Comprehensive Cancer Center Biostatistics and Informatics Shared Resource (supported by NCI P30 CA125123). This project was also supported by the Cytometry and Cell Sorting Core at Baylor College of Medicine with funding from the NIH (P30 AI036211, P30 CA125123, and S10 RR024574) and the kind assistance of Joel M. Sederstrom. We thank Dr Joe W. Gray for providing the genomic data for breast cancer cell lines, Dr Susan G. Hilsenbeck for the helpful discussion on biostatistics, Dr Gary C. Chamness for editing this manuscript, Ms. Fuli Jia, Ms. Myra Grace Costello and Dr Kimberley Holloway for their technical assistance in RPPA experiments, and Dr Kimal Rajapakshe, Cristian Coarfa, and Qianxing Mo for RPPA data processing and normalization. RPPA analysis was supported in part by Cancer Prevention \& Research Institute of Texas Proteomics \& Metabolomics Core Facility Support Award (RP120092) (D.P.E. and S.H.) and NCI Cancer Center Support Grant to Antibody-based Proteomics Core/Shared Resource (P30CA125123) (D.P.E. and S.H.). We would like to acknowledge the Molecular Cytogenetics Facility, Center for Genetics and Genomics, The University of Texas M.D. Anderson Cancer Center, Houston, Texas for FISH analysis.

\section{Author contributions}

X.-S.W. supervised the study, performed bioinformatics analysis, analysed the data, and wrote the manuscript. J.A.K. designed and performed cell biology, mechanistic, and in vivo experiments, analysed the data and wrote the manuscript. Y.T. designed and 
performed molecular biology experiments. X.W. assisted the in vivo studies and RPPA analysis. X.C. and J.V. assisted the cell biology experiments. Y.L. advised on the cell biology experiments. S.H. and D.P.E. provided collaboration on RPPA experiments. X.P. provided the inducible vector. R.S. and K.L. advised on the study and revised the manuscript.

\section{Additional information}

Supplementary Information accompanies this paper at http://www.nature.com/ naturecommunications

Competing financial interests: The authors declare no competing financial interests.

Reprints and permission information is available online at http://npg.nature.com/ reprintsandpermissions/
How to cite this article: Kim, J. A. et al. Comprehensive functional analysis of the tousled-like kinase 2 frequently amplified in aggressive luminal breast cancers. Nat. Commun. 7, 12991 doi: 10.1038/ncomms12991 (2016).

cc (i) This work is licensed under a Creative Commons Attribution 4.0 International License. The images or other third party material in this article are included in the article's Creative Commons license, unless indicated otherwise in the credit line; if the material is not included under the Creative Commons license, users will need to obtain permission from the license holder to reproduce the material. To view a copy of this license, visit http://creativecommons.org/licenses/by/4.0/

(C) The Author(s) 2016 